\title{
DESENVOLVIMENTO DE ALGORITMO COMPUTACIONAL PARA VOLUMETRIA DE ESTRUTURAS CEREBRAIS EM IMAGENS DE RESSONÂNCIA MAGNÉTICA NUCLEAR
}

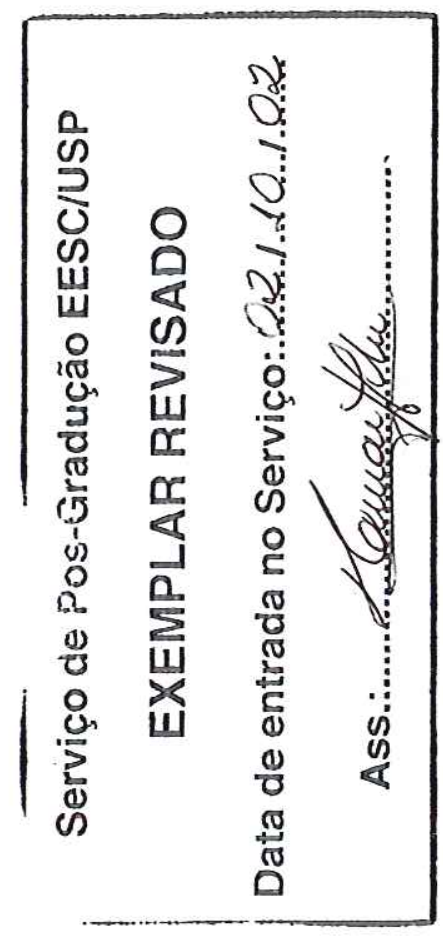

\section{Luciene Cavalcanti Rodrigues}

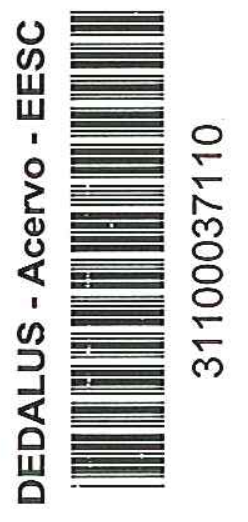

Dissertação apresentada à Escola de Engenharia de São Carlos da Universidade de São Paulo, como parte dos requisitos para obtenção do título de Mestre em Engenharia Elétrica.

Orientador: Prof. Dr. Paulo Mazzoncini de Azevedo Marques

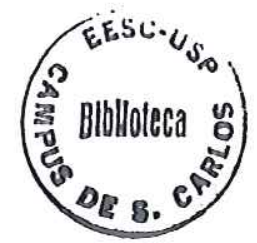

São Carlos, 2002 
curs. 19915

Tombo TO259102

Sysno $12+7023$

Ficha catalográfica preparada pela Seção de Tratamento da Informação do Serviço de Biblioteca - EESC/USP

R696d

Rodrigues, Luciene Cavalcanti

Desenvolvimento de algoritmo computacional para volumetria de estruturas cerebrais em imagens de ressonância magnética nuclear / Luciene Cavalcanti Rodrigues. -- São Carlos, 2002.

Dissertação (Mestrado) -- Escola de Engenharia de São Carlos-Universidade de São Paulo, 2002

Área : Engenharia Elétrica.

Orientador: Prof. Dr. Paulo Mazzoncini de Azevedo Marques.

1. Volumetria. 2. Epilepsia. 3. Ressonância magnética nuclear. 4. Processamento de imagens. I. Título. 
FOLHA DE JULGAMENTO

Candidata: Tecnóloga LUCIENE CAVALCANTI RODRIGUES

Dissertação defendida e julgada em 12-08-2002 perante a Comissão Julgadora:
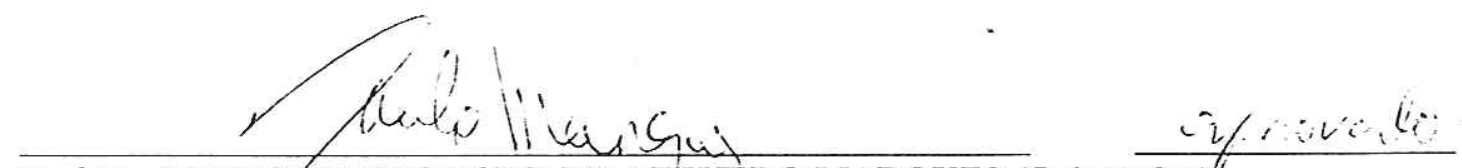

Prof. Dr. PAULOMAZZONCINI DE AZEVEDO MARQUES (Orientador)"

(Faculdade de/Medicina/USP)

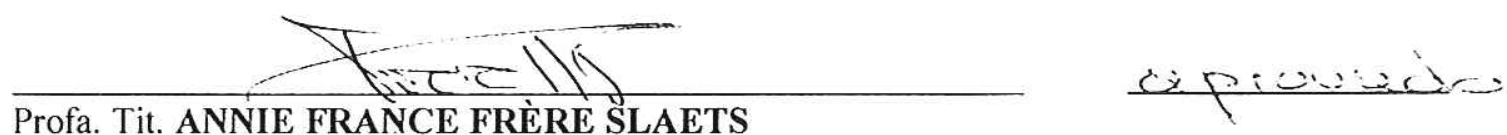

(Escola de Engenharia de São Carlos/USP)

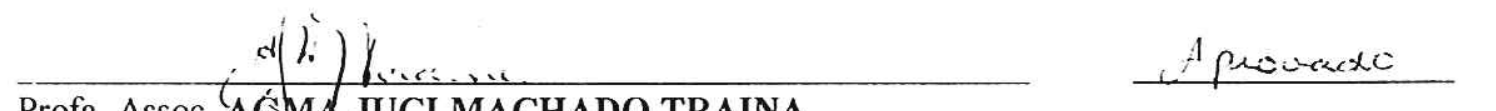

Profa. Assoc. AǴMA JUCI MACHADO TRAINA

(Instituto de Ciências Matemáticas e de Computação/USP)

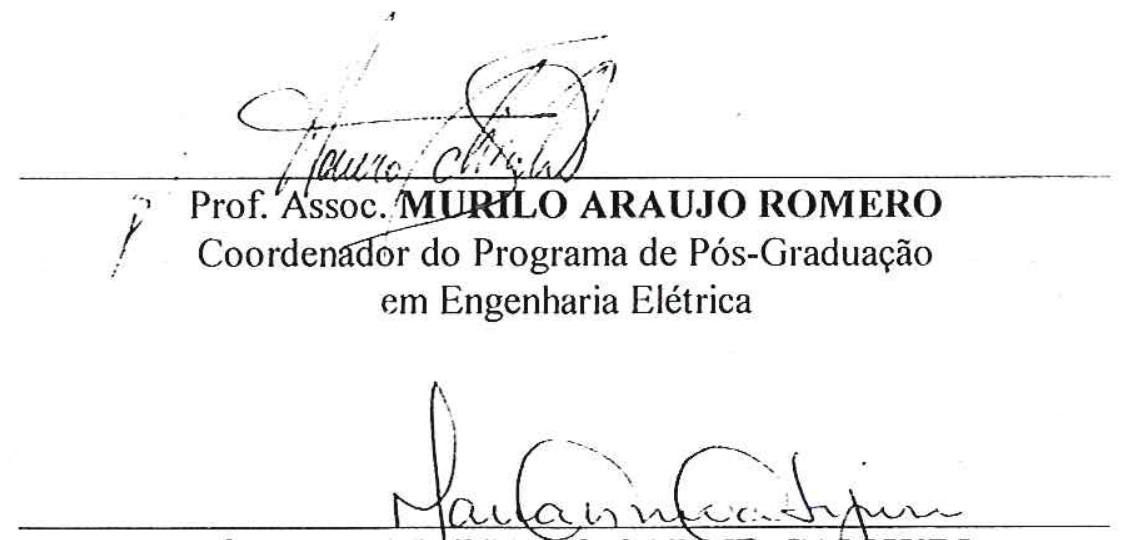

Profa. Assoc. MARIA DO CARMO CALJJURI

Presidente dalComissão de Pós-Graduação 
"É muito melhor arriscar coisas grandiosas,

e alcançar triunfo e glória mesmo expondo-se à derrotas,

do que formar fila com os pobres de espírito que

nem gozam muito nem ofrem muito,

porque vivem nessa penumbra cinzenta que não conhece

vitória nem derrota ..."

"As pessoas que vencem neste mundo são as que procuram as circunstâncias de que precisam e quando não as encontram, as criam..."

"Há homens que lutam um dia e são bons, Há homens que lutam um ano e são melhores, Há os que lutam muitos anos e são muito bons, Mas há os que lutam toda a vida, esses são imprescindíveis ..." 
A Deus, pela vida, saúde, paz e grande oportunidade de realizar este trabalho.

Aos amigos espirituais que sempre guiam meus passos e me trazem a paz e tranquilidade por vezes perdida.

A minha mãe, pelo amor, carinho, dedicação, compreensão e por sempre estar presente em todos os momentos da minha vida.

Aos meus irmãos, que mesmo não compreendendo o trabalho sempre se interessaram, apoiaram minha caminhada e tiveram paciência. 
Acredito que um simples agradecimento aqui deixado jamais demonstrará o grau de importância que essas pessoas são em minha vida e em meus caminhos. Agradeço aos ex-professores e amigos Valéria Maria Volpe e Márcio Alexandre Marques pelo apoio e confiança no ingresso nesta caminhada, ao professor e amigo Paulo Mazzoncini de Azevedo Marques pela gratificante orientação, convívio e enorme paciência dispensada para que os "meios" chegassem aos "fins", e a Dra. Luciana Torres Ribeiro pela presença decisiva na conclusão deste trabalho.

"Se não houver frutos, valeu a beleza das flores, Se não houver flores, valeu a sombra das folhas, Se não houver folhas, valeu a intenção da semente." 
À professora Annie France Frére Slaets, pelo apoio e colaboração em todos os momentos.

Aos ex-professores e amigos Djalma Domingos, Carlos Magnus, Luis Paulo, entre outros, que participaram de minha formação acadêmica e incentivaram a realização deste trabalho.

Aos amigos do CHOPI: André, David, Edilson, Lucas (chefe), Marcelo Costa (Puff), Marcelo (Honda) e Roberto (Chico), pelo apoio, companheirismo e momentos descontraídos que passei com eles.

Aos amigos do @ladim: Clayton, Flávio, Leandro, Marcelo, Silvia, Vivian e os demais integrantes do laboratório pela amizade.

A todos os amigos, presentes ou ausentes, que acompanharam de perto ou à distância esta caminhada e torceram para que tudo desse certo.

Ao Departamento de Engenharia Elétrica da Escola de Engenharia de São Carlos da Universidade de São Paulo, pela oportunidade de realização deste trabalho.

Aos docentes e funcionários do Centro de Ciências das Imagens e Física Médica da Faculdade de Medicina de Ribeirão Preto da Universidade de São Paulo, pelo apoio e espaço para o desenvolvimento deste trabalho.

À Fundação de Amparo a Pesquisa do Estado de São Paulo (FAPESP), pelo apoio financeiro, processo $n^{\circ} 99 / 10545-5$. 
Palavras-chaves: Volumetria, Epilepsia, Ressonância Magnética Nuclear,

Processamento de Imagens.

\section{RESUMO}

Este projeto apresenta a pesquisa e desenvolvimento de um algoritmo computacional para realizar a avaliação volumétrica de estruturas cerebrais, a partir de imagens de Ressonância Magnética Nuclear (RMN). O algoritmo possibilita a seleção das estruturas a serem avaliadas nos vários planos do exame e, então realiza a integração das áreas calculadas para cada corte de modo a obter o volume de cada estrutura selecionada. O contorno da região de interesse em cada corte é definido pelo médico especialista de forma interativa, através da combinação de "tresholding" com uma função de edição manual de contorno.

Os resultados obtidos indicaram a potencialidade de uso do algoritmo para o auxílio ao diagnóstico e estadiamento clínico de doenças como Mal de Alzheimer e Epilepsia do lobo temporal. O algoritmo foi considerado suficientemente preciso pelos especialistas que o testaram, mantendo a variação das medidas de volume abaixo de 1,5 $\mathrm{mm}^{3}$. O tempo médio para a realização de um estudo completo foi de aproximadamente 25 minutos. 
Keywords: Volumetry, Epilepsy, Nuclear

Magnetic Ressonance, Imaging Processing.

\begin{abstract}
This project presents the development of a computational algorithm focused on the volumetric measurement of cerebral structures in Nuclear Magnetic Resonance Images. The algorithm allows the selection of structures to be analysed on each exam slices and realizes the integration of calculated areas for each slice to get a volumetric measurement of each selected structure. The edge of interest region in each slice must be interatively defined for a specialist, based on a combination of thresholding with a manual segmentation function.
\end{abstract}

The results points to the potenciality of the algorithm aplication to aid the diagnosis of diseases as Alzheimer's disease and Temporal Lobe Epilepsy. 
Este trabalho teve o apoio financeiro da FAPESP - Fundação de Amparo à Pesquisa do Estado de São Paulo - Processo n ${ }^{\circ}$ 99/10545-5. 


\section{SUMÁRIO}

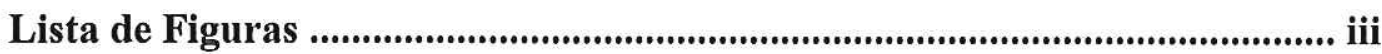

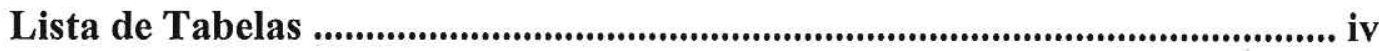

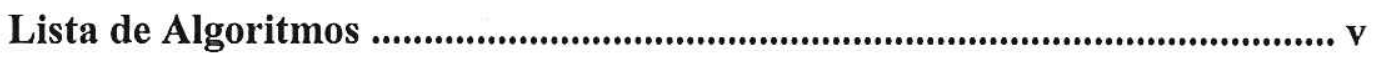

Capítulo 1 - Introdução ............................................................................................ 1

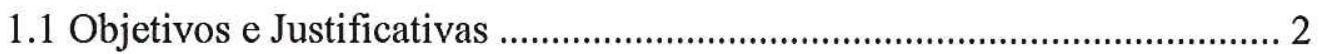

1.2 Organização da Dissertação ................................................................ 4

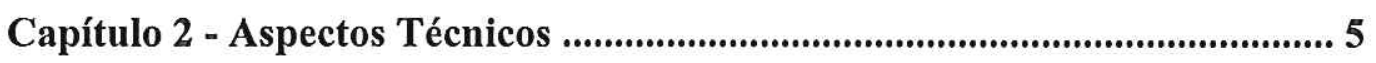

2.1 Métodos de Neuroimagem .............................................................. 5

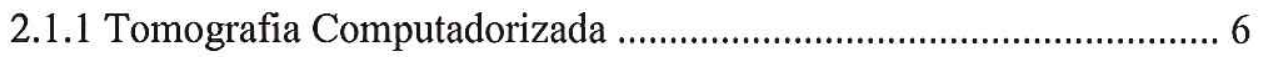

2.1.2 Ressonância Magnética Nuclear ................................................ 8

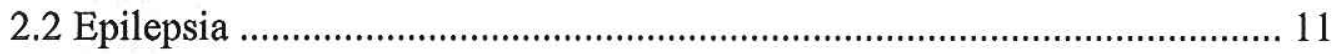

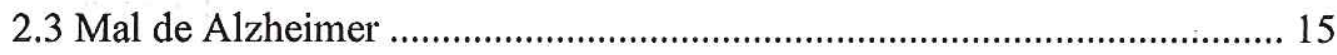

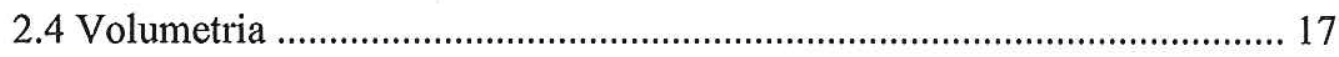

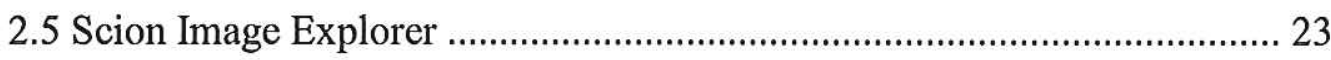

Capítulo 3 - Padrão DICOM 3.0 ..................................................................... 27

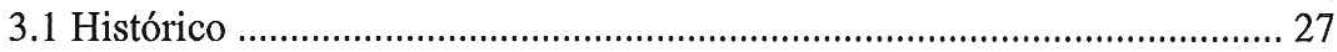

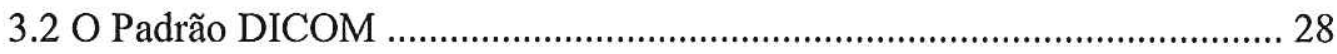

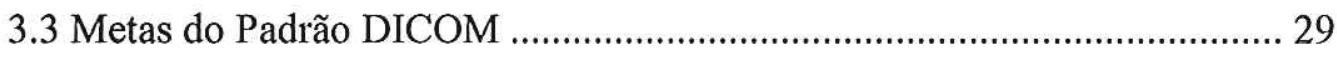

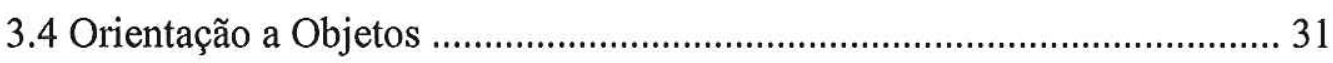

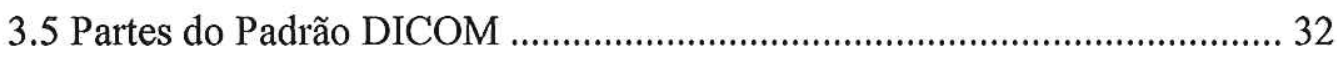

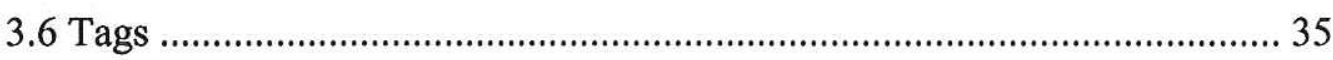

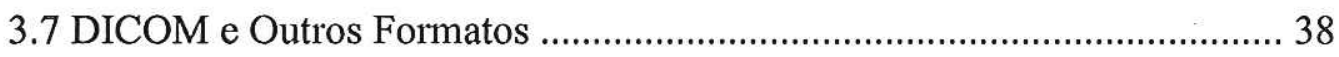


Capítulo 4 - Materiais e Métodos ............................................................. 40

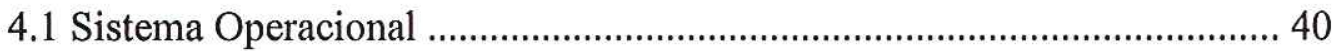

4.2 Linguagem para Desenvolvimento ......................................................... 42

4.3 Software Desenvolvido .......................................................................... 44

4.4 Metodologia para Avaliação do Software ............................................... 48

Capítulo 5 - Resultados Obtidos .........................................................................48

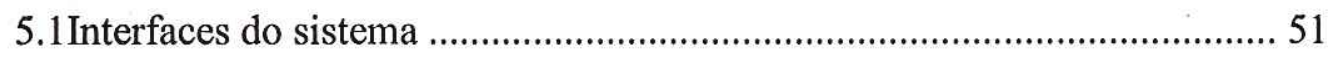

5.2Avaliação qualitativa ........................................................................... 53

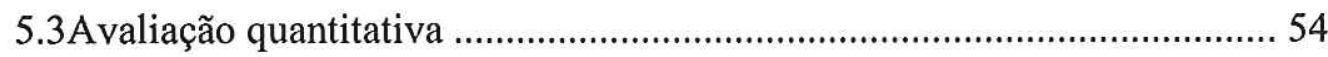

Capítulo 6 - Discussão dos Resultados e Conclusões ......................................... 56

Capítulo 7 - Referências Bibliográficas ............................................................. 61

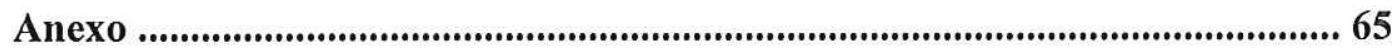




\section{LISTA DE FIGURAS}

Figura 2.1 - Estruturas importantes para a morfometria cerebral ...................... 10

Figura 2.2 - Scion Image em plataforma Macintosh ...................................... 24

Figura 2.3 - Scion Image em plataforma Windows .......................................... 25

Figura 3.1 - Processo de construção para uma declaração de conformidade ....... 31

Figura 3.2 - Partes atuais do padrão DICOM e partes propostas para extensão

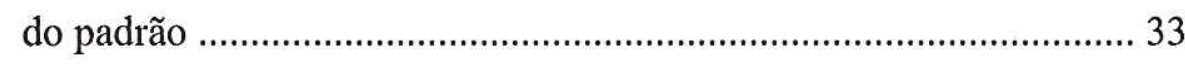

Figura 5.1. Janela Principal do software ..................................................... 51

Figura 5.2. Interface para escolha do diretório .............................................. 52

Figura 5.3. Interface com a primeira imagem ….......................................... 52

Figura 5.4. Interface com algumas estruturas de interesse marcadas ................. 53

Figura 6.1 - Reconstrução do Hipocampo ........................................................... 59

Figura 6.2 - Módulo de Co-registro: Imagem de RM e de SPECT com marcadores

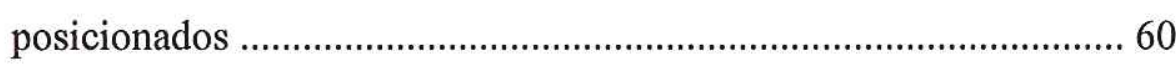




\section{LISTA DE TABELAS}

Tabela 2.1 - Normalização das medidas volumétricas da formação hipocampal (FH) e amígdala (AM) em Ressonância Magnética Nuclear 20

Tabela 3.1 - Resumo das partes do padrão DICOM 3.0 ([FREIRE-1997]) .......... 35

Tabela 3.2 - Tags do grupo 0010 - Informações sobre o Paciente ....................... 36

Tabela 3.3 - Tags reconhecidas e utilizadas para o trabalho ............................... 37

Tabela 3.4 - Tags reconhecidas e com seus respectivos valores .......................... 38

Tabela 4.1 - Base de cálculo para a assimetria e os valores normalizados ......... 47

Tabela 4.2 - Resultados mostrados ao usuário após o término do estudo ........... 48

Tabela 4.3 - Valores calculados pelo sistema a partir das marcações das

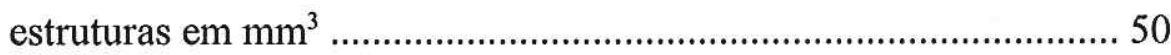

Tabela 5.1 - Valores para a avaliação da interface ............................................ 54

Tabela 5.2 - Resultados comparativos obtidos através do sistema em $\mathrm{mm}^{3}$........ 55 


\section{LISTA DE ALGORITMOS}

Algoritmo 1 - Algoritmo para organização, abertura e visualização das imagens 45

Algoritmo 2 - Cálculo do volume de acordo com o princípio de Cavalieri 47 


\section{Capítulo 1}

\section{Introdução}

Várias pesquisas indicam que o lobo temporal apresenta variações de volume associadas com alguns tipos de doenças, tais como esquizofrenia, epilepsia, síndrome velocardiofacial, entre outras. O Mal de Alzheimer, por exemplo, é caracterizado pelo declínio cognitivo e atrofia cerebral progressiva. A atrofia é uma conseqüência de perdas celulares que pode ser visualizada em vida utilizando-se tomografia ou ressonância magnética nuclear ([CENDES-1995]). A esclerose hipocampal, por outro lado, é a patologia mais comum encontrada em pacientes com epilepsia do lobo temporal e a volumetria hipocampal pode fornecer uma medida direta da atrofia do hipocampo associada com a esclerose hipocampal.

Nos últimos anos, medidas da formação do hipocampo em imagens de ressonância magnética volumétrica de alta resolução tem sido utilizadas na avaliação pré-operatória de pacientes com epilepsia do lobo temporal unilateral ([CIESIELSKI1999], [COOK-1992]). As imagens de ressonância magnética nuclear volumétricas, quando utilizadas em conjunto com a eletroencefalografia monitorada em vídeo, estudos neuropsicológicos e outros estudos de neuroimagens, possibilitam aos pacientes com epilepsia do lobo temporal serem tratados de forma mais apropriada e eficiente ([COOK-1992]). 
Esses fatores, juntamente com a necessidade dos profissionais da área, tem levado ao direcionamento de várias pesquisas para o desenvolvimento de métodos eficazes para morfometria de estruturas cerebrais de forma objetiva e reprodutível.

\subsection{Objetivos e Justificativas}

O Hospital das Clínicas realiza cerca de 720 exames de Ressonância Magnética Nuclear por ano com a finalidade de estudar o comportamento patológico de pacientes epiléticos, que consequentemente podem fazer parte das cerca de 80 cirurgias de epilepsia que são realizadas anualmente pelo CIREP (Centro de Cirurgia de Epilepsia). Com os exames em mãos o médico realiza a análise visual das imagens, tentando encontrar possíveis assimetrias lateralizadas ou não para posicionar anatomicamente o foco epiléptico do paciente. O médico é também responsável por quantificar a assimetria dos pacientes epiléticos através do cálculo do volume das estruturas cerebrais.

Anteriormente ao desenvolvimento deste trabalho, o médico utilizava um software chamado Scion Image Explorer, que nada mais é do que um software de processamento de imagens e não algo específico para análises volumétricas. O diferencial deste software é que ele possui uma ferramenta para marcação de regiões de interesse nas imagens e efetua o cálculo da área desta região marcada. Porém o trabalho do médico era exaustivo, visto que a quantidade de imagens de um exame completo de Ressonância Magnética Nuclear pode variar de 1 a 200, por exemplo, dependendo do tamanho da cabeça do paciente e do protocolo utilizado e o médico tinha que efetuar a marcação em cada uma das imagens e transferir o valor da área das regiões para uma 
planilha eletrônica', que fazia os cálculos necessários. Neste processo, o médico especialista levava cerca de duas horas para obter o resultado final do estudo para um único paciente.

Embora existam produtos comercialmente disponíveis e aplicáveis à volumetria de estruturas cerebrais, de modo geral, além de seu custo elevado existem limitações em sua aplicação relacionadas às particularidades de cada serviço, especialmente ao que se refere à interação com o usuário e às funções básicas implementadas. Por esses motivos, os principais centros internacionais de avaliação neurológica possuem soluções computacionais localmente desenvolvidas para esse fim. Neste contexto, o objetivo principal desta pesquisa foi o desenvolvimento de um algoritmo computacional para volumetria de estruturas cerebrais, visando o auxílio computadorizado ao diagnóstico e avaliação clínica da epilepsia do lobo temporal, que possuísse características otimizadas em relação aos serviços do Hospital das Clínicas da Faculdade de Medicina de Ribeirão Preto, fosse de baixo custo e desenvolvido sobre uma filosofia de plataforma open-source.

É importante realçar que embora possuindo uma aplicação bem definida, o algoritmo desenvolvido poderá ser utilizado em outras pesquisas que necessitem de procedimentos de volumetria de estruturas a partir de imagens de Ressonância Magnética Nuclear.

1 Ver anexo planilha utilizada. (Dr. David Araújo) 


\subsection{Organização da Dissertação}

Este trabalho está dividido em 7 capítulos, sendo que o introdutório traz uma abordagem geral da importância da volumetria de estruturas cerebrais no auxílio ao diagnóstico e localização de focos epilépticos, bem como os objetivos e justificativas desta pesquisa.

O capítulo 2 apresenta considerações sobre aspectos técnicos estudados para elaboração do trabalho, tais como Anatomia Cerebral, Epilepsia, Mal de Alzheimer, Volumetria e o Scion Image Explorer.

O capítulo 3 descreve as características do padrão DICOM 3.0, que é o padrão de imagens adotado pelos aparelhos de Ressonância Magnética Nuclear.

No capítulo 4 discute-se os materiais e métodos escolhidos e as características do software desenvolvido.

O capítulo 5 apresenta os resultados obtidos.

No capítulo 6 são discutidos os resultados obtidos e apresentadas as conclusões do estudo realizado.

Finalmente, no capítulo 7 são apresentadas as referências bibliográficas. 
Capítulo 2

Aspectos Técnicos

\subsection{Métodos de Neuroimagem}

As técnicas modernas de neuroimagem estrutural têm aumentado dramaticamente a capacidade de avaliação de pacientes com epilepsia intratável. Nas últimas duas décadas, os métodos que forneciam apenas evidências indiretas de uma anormalidade como, por exemplo, a radiografia do crânio, foram substituídos por modalidades que resultam na visualização direta da anatomia, como a Tomografia Computadorizada e a Ressonância Magnética Nuclear. Atualmente, como resultado destes avanços, é possível examinar rotineiramente o cérebro de um paciente com epilepsia com risco pequeno e uma sensibilidade diagnóstica aumentada.

Antes do início da década de 70, as imagens cerebrais eram obtidas por raios X do crânio, arteriografia, pneumoencefalografia e estudos com radioisótopos. Os raios $\mathrm{X}$ do crânio, embora baratos e facilmente obtidos, mostram uma anormalidade específica em pouquíssimos pacientes com epilepsia parcial e não são mais utilizados para propósitos diagnósticos. As indicações atuais destes estudos na avaliação da epilepsia incluem a verificação da viabilidade do retalho ósseo após craniotomia para 
cirurgia de epilepsia e a determinação da posição de eletrodos intracranianos. A arteriografia também tem apresentado um papel menor na avaliação e terapêutica de pacientes com epilepsia, sendo associada com morbidade pequena mas significativa, incluindo acidentes vasculares e reações ao contraste iodado. Este procedimento atualmente é realizado na avaliação para cirurgia de epilepsia com a finalidade de obtenção de informação sobre a anatomia vascular antes da ressecção cirúrgica.

A Ressonância Magnética Nuclear é atualmente a principal técnica de neuroimagem utilizada na avaliação de pacientes com epilepsia nos Estados Unidos e na maioria dos países desenvolvidos. No entanto, esta técnica pode não estar disponível facilmente em alguns países em desenvolvimento e a Tomografia Computadorizada é, nestas situações, o método de imagem mais importante. O propósito dos estudos de neuroimagem estrutural na avaliação de pacientes com epilepsia parcial é estabelecer a etiologia da desordem crítica e auxiliar os estudos eletrofisiológicos na localização da zona epileptogênica. Deve ser ressaltado que a informação fornecida pela neuroimagem estrutural complementa os dados eletrofisiológicos, e a presença de uma lesão estrutural pode não necessariamente implicar em epileptogenicidade.

\subsubsection{Tomografia Computadorizada}

A importância da Tomografia Computadorizada em pacientes com epilepsia parcial intratável foi verificada logo após a introdução desta técnica no final da década de 70. As anormalidades intraparenquimatosas associadas com epilepsia parcial 
podem ser visualizadas diretamente pela Tomografia Computadorizada, particularmente as grandes neoplasias primárias intracanianas e as malformações vasculares. Além disso, a Tomografia Computadorizada é muito sensível na demonstração das hemorragias intraparenquimatosas e das calcificações associadas a lesões epileptogênicas. A sensibilidade da Tomografia Computadorizada pode ser aumentada pela injeção de um agente contrastante rádio-opaco que aumenta a radiodensidade de algumas lesões, incluindo as anormalidades vasculares e as lesões nas quais ocorre ruptura da barreira hematoencafálica ([SUSAN-1998]).

Embora os aparelhos de Tomografia Computadorizada sejam mais facilmente disponíveis na maioria dos centros e as imagens possam ser obtidas rapidamente e facilmente interpretadas, as imagens por Tomografia Computadorizada não possuem outras vantagens sobre as da Ressonância Magnética Nuclear. Porém, como o tempo para a obtenção da Tomografia Computadorizada é de somente 5 a 10 minutos para imagens reconstruídas no plano axial, a Tomografia Computadorizada é o método de investigação de escolha em pacientes com crises agudas, nos quais se suspeita de uma lesão que coloca a vida em risco, sendo necessário diagnóstico rápido. As desvantagens das imagens por Tomografia Computadorizada incluem a dificuldade para a obtenção de imagens multiplanares, as reações do indivíduo ao agente contrastante, a visualização inadequada dos lobos temporais em decorrência de artefatos ósseos da fossa média e a exposição à radiação. Na avaliação diagnóstica de epilepsia crônica, a Tomografia Computadorizada tem se mostrado insensível para a detecção de substratos patológicos pequenos, como as displasias corticais focais e a esclerose mesial temporal. Contudo, a despeito destes problemas, a Tomografia Computadorizada ainda representa um método diagnóstico valioso, particularmente nos locais onde não existe 
Ressonância Magnética Nuclear. Em pacientes com tumores grandes ou outras lesões estruturais óbvias, a Tomografia Computadorizada e a Ressonância Magnética Nuclear podem delinear igualmente bem a lesão; além disso, a Tomografia Computadorizada pode complementar a Ressonância Magnética Nuclear em situações onde estão presentes lesões ósseas ou anormalidades intracranianas calcificadas ([SUSAN-1998]).

\subsubsection{Ressonância Magnética Nuclear}

A Ressonância Magnética Nuclear é atualmente a técnica de neuroimagem mais importante para a avaliação pré-operatória de pacientes com epilepsia parcial intratável. Sua superioridade sobre a Tomografia Computadorizada é bem estabelecida. As vantagens da Ressonância Magnética Nuclear incluem dados anatômicos multiplanares excelentes, sem artefatos ósseos e a ausência de radiação ionizante. Ela acarreta risco mínimo, como reação de confinamento em pacientes claustrofóbicos e, raramente, reações alérgicas ou toxicidade cordiorrenal ao agente de contraste ([SUSAN-1998]).

Considerando-se os métodos de neuroimagem estrutural, a ressonância magnética é mais sensível do que a tomografia computadorizada para a avaliação das estruturas têmporo mesiais, constituindo-se no método de escolha em portadores de epilepsias refratárias ao tratamento clínico e estudo de pacientes com mal de Alzheimer, pois, além de permitir a detecção de causas lesionais ou evidências de tecido anômalo, evidencia os achados característicos da esclerose temporal mesial, em candidatos a 
lobectomia temporal. ([ROGACHESKI-1998])

Por ter excelente contraste em tecido mole e pelo fato de ser virtualmente não-invasiva é particularmente boa para estudos envolvendo órgãos tais como o cérebro ([BALLESTER-2000]), e, por isso, a imagem por ressonância magnética tornou-se uma das mais importantes modalidades de imagens em estudos neurológicos.

O protocolo de Ressonância Magnética Nuclear ou as estratégias de imagem para pacientes epilépticos podem variar entre instituições diferentes. Com o decorrer dos anos surgiram técnicas diferentes, algumas das quais estão ainda sendo testadas. Em geral, as seqüências ponderadas em T2, com tempo de repetição/tempo de eco longo (TR/TE), são preferidas para a detecção de lesões patológicas focais enquanto as seqüências ponderadas em T1, com TR/TE curto, delineiam mais distintamente as relações anatômicas. Segundo [SUSAN-1988], o protocolo mais importante usado é a seqüência ponderada em T1, aquisição volumétrica, na técnica de gradiente eco, em seqüência de cortes contíguos finos $(1,5 \mathrm{~mm})$, suplementada pelas seqüências ponderadas em T2. As imagens são adquiridas no plano coronal, perpendicular ao eixo ao longo do hipocampo. As vantagens das imagens coronais incluem o melhor delineamento da anatomia mesial temporal e as secções finas também aumentam a sensibilidade. Dependendo da indicação clínica, utilizam-se protocolos adicionais como “inversion recovery" (IR), seqüências "fluid attenuation inversion recovery (FLAIR), relaxometria em T2 e volumetria hipocampal, as quais têm o potencial de aumentar ainda mais a sensibilidade da Ressonância Magnética Nuclear na detecção das lesões epileptogênicas. Os estudos contrastados com gadolíneo-DTPA não são usados rotineiramente, a menos que seja detectada uma lesão expansiva com os protocolos rotineiros. 
Atualmente a perda neuronal em esclerose mesial temporal pode ser demonstrada nos métodos de neuroimagem, sobretudo pela ressonância magnética, devido a atrofia e alteração da morfologia interna, associadas a proliferação gliótica, que se traduz por hipointensidade em T1 e hiperintensidade em T2 nas imagens ([ROGACHESKI-1998], [WEBB-1999]). A figura 2.1 mostra um exemplo de imagem de Ressonância Magnética com algumas estruturas cerebrais destacadas.

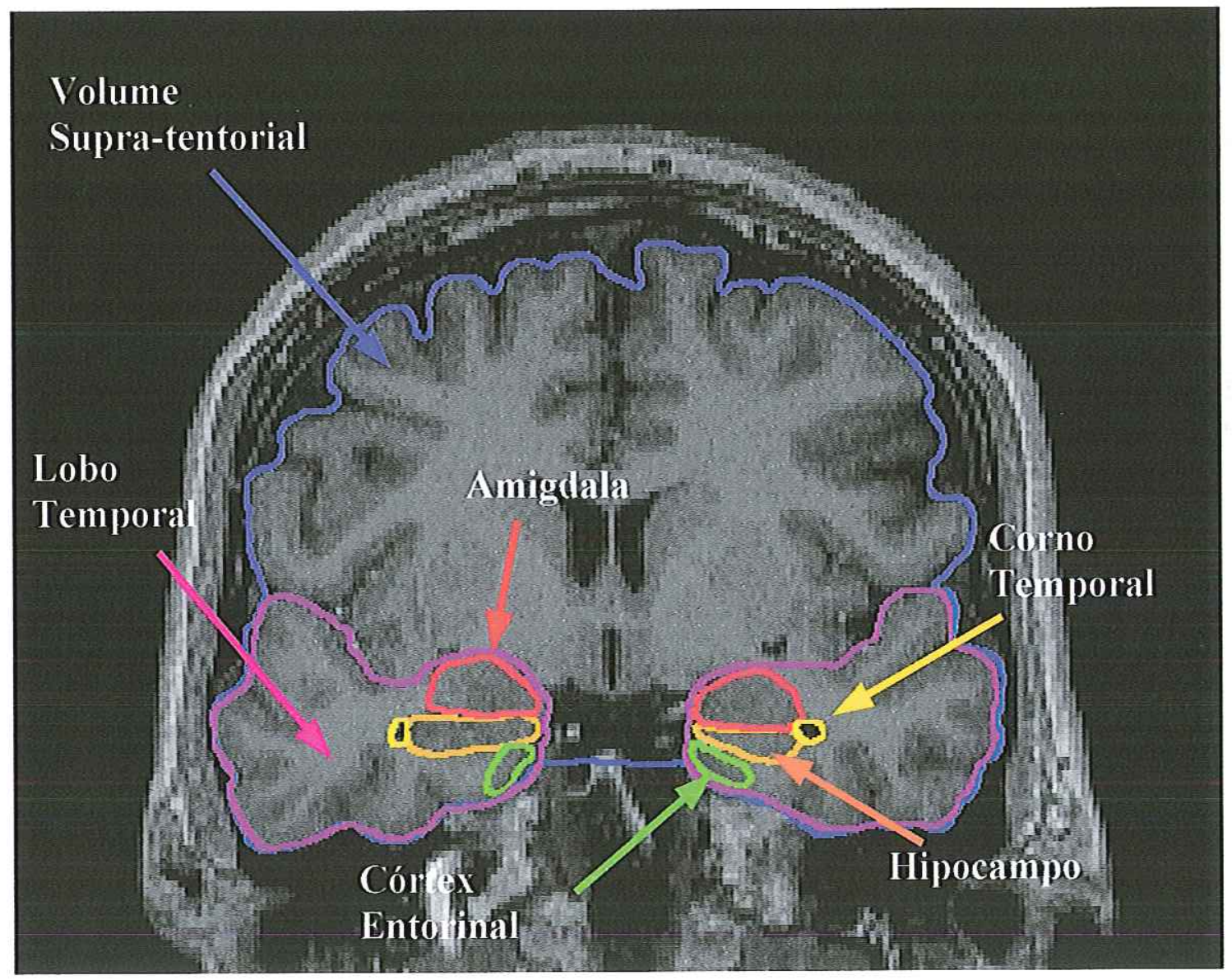

Figura 2.1 - Estruturas importantes para a morfometria cerebral 


\subsection{Epilepsia}

A avaliação pré-cirúrgica tradicional utiliza-se da vídeoEletroencefalografia na fase inicial para decidir se o paciente é um bom candidato cirúrgico. Uma das limitações desta forma de avaliação é o fato de que muitos pacientes demonstram, durante as crises, atividade eletroencefalográfica bilateral ou artefato muscular que obscurece a Eletroencefalografia.

Segundo [WEBB-1999] a epilepsia é a mais predominante desordem neurológica crônica, sendo mais comumente originária do lobo temporal mesial.

A esclerose hipocampal é a patologia mais comum encontrada em pacientes com epilepsia do lobo temporal, estando presente em aproximadamente $60 \%$ a $75 \%$ dos pacientes sob tratamento cirúrgico ([CIESIELSKI-1999]), que são pacientes que têm crises resistentes ao tratamento clínico e que correspondem de $10 \%$ a $30 \%$ do total de casos ([ROGACHESKI-1998]).

O hipocampo é uma estrutura complexa do lobo temporal mesial e faz parte do sistema límbico e do circuito da memória, sendo o foco principal da epilepsia do lobo temporal e também afetado no início do mal de Alzheimer. Por ser uma estrutura visível pela ressonância magnética, sua avaliação pode ser importante, não só em epilepsia, mas também no mal de Alzheimer, esquizofrenia e outras doenças amnésticas, através da medida do seu volume ([ROGACHESKI-1998], [LAAKSO1997]).

Existe uma forte relação entre o grau de perda de volume do hipocampo e a severidade da esclerose hipocampal, detectada em estudos utilizando técnicas 
neuropatológicas qualitativas, semiquantitativas e quantitativas. Diante desses achados, aparentemente a severidade da esclerose hipocampal pode ser adequadamente prevista a partir da medida dos volumes e razões de volume dos hipocampos. Essa informação pode ser útil no diagnóstico, terapêutica, prognóstico e investigações científicas sobre o tratamento de pacientes com epilepsia do lobo temporal não tratável com medicação ([COOK-1992],[WATSON-1997],[LAAKSO-1997],[INSAUSTI-1998]).

Muitos estudos tem demonstrado que a imagem por ressonância magnética é a técnica pré-operatória mais confiável na detecção de esclerose temporal mesial ([KUZNIECKY -1997]), sendo a deteç̧ão quantitativa da atrofia hipocampal em imagens de ressonância magnética e sua correlação com a esclerose hipocampal comprovada patologicamente em adultos com epilepsia do lobo temporal mesial ([ELIEZ-2000], [ROVARIS-1999]), [INSAUSTI-1998]).

Quando a atrofia hipocampal é unilateral pode ser inequivocamente identificada em imagens de ressonância magnética, apresentando uma situação de provável epilepsia temporal focal. Por outro lado, a prevalência de assimetria hipocampal é muito baixa em adultos com epilepsia extratemporal ([LAWSON-1997], [FOX-2000]), e a especificidade da assimetria hipocampal para a síndrome de esclerose do lobo temporal mesial tem sido comprovada de forma intensa ([LAWSON-1997]).

Muitos estudos tem sido feitos com grupos de pacientes com crises originadas em estruturas extra-temporais e extra-hipocampais utilizando imagens de ressonância magnética nuclear de forma a se tentar determinar se essas crises estão também associadas com perdas de células e subsequente atrofia e perda de volume no hipocampo e amigdala ([CENDES-1995], [WATSON-1997]). Alguns estudos 
mostraram a não redução do volume hipocampal em pacientes com epilepsia de longa duração causada por lesões em estruturas extra-temporais e outros encontraram uma baixa incidência (6\%) de "dual pathology", uma condição na qual o paciente apresenta as duas possibilidades de epilepsia. De modo geral, nenhuma redução de volume hipocampal ou amigdalóide foi encontrada em estudos envolvendo pacientes com epilepsia generalizada primária de longa duração e epilepsia com generalização secundária. Portanto, reduções significativas nos volumes hipocampais parecem configurar um marcador específico para a esclerose hipocampal, possibilitando o uso de imagens volumétricas de ressonância magnética na diferenciação entre pacientes com epilepsia do lobo temporal e epilepsia extra-temporal ([CENDES-1995], [WATSON1995], [WATSON-1996]).

O binômio atrofia hipocampal-aumento de sinal em T2 no interior da formação hipocampal, constituiu, durante muito tempo, a chave para o diagnóstico da esclerose temporal mesial, através de inspeção visual. Recentemente, descreveu-se também a redução de sinal, como indicativo de gliose, em cortes finos $(5 \mathrm{~mm})$ das formações hipocampais obtidos com técnica inversion-recovery. Enfatiza-se também um grau variável de achatamento do hipocampo como sinal indicativo de alteração morfológica associado à atrofia hipocampal ([ROGACHESKI-1998]).

Técnicas especializadas de ressonância magnética para a avaliação da integridade hipocampal são utilizadas como um componente da avaliação pré-cirúrgica. Em particular, o volume hipocampal deve ser estimado utilizando-se o método de Cavalieri, no qual uma estimativa do volume das estruturas é obtida pela multiplicação da soma das áreas das seções da estrutura nas imagens consecutivas de ressonância magnética pelo intervalo da seção. De modo geral, técnicas manuais para estimativas do 
volume do hipocampo são sensíveis e específicas para a detecção da atrofia hipocampal, porém altamente especializadas. Técnicas para segmentação automática também têm sido pesquisadas ([WEBB-1999]).

Em [JACK JR.-1992a] foi desenvolvida a proposta de uma técnica volumétrica baseada em ressonância magnética e conseguiram reprodutibilidade e validade em vários conjuntos clínicos e pesquisas. Esta técnica tem sido utilizada em medidas de volume da formação hipocampal de pacientes que estão sendo avaliados para cirurgia e uma excelente correspondência tem sido encontrada entre a atrofia da formação hipocampal, como avaliado pela ressonância magnética, e o local da ativação do foco epileptogênico. Além disso, um estudo correlacionando volumes de ressonância magnética da formação hipocampal com o grau de esclerose mesial, encontrada em espécimes cirúrgicas de pacientes epiléticos, demonstrou excelente concordância entre a atrofia vista em ressonância magnética e a perda neuronal na formação hipocampal. Neste trabalho citado, o processamento da imagem foi feito com o software ANALYSE (Biodynamics Research Unit, Mayo Foundation, Rochester, MN), que aplica uma técnica semiautomática que combina "tracing" e "thresholding".

Em [JACK JR.-1992b] foi analisado o relacionamento entre volumes da formação hipocampal e o resultado depois da lobectomia anterior temporal (isto é, controle pós-operatório) para epilepsia intratável em 50 pacientes consecutivos dos quais as amostras cirúrgicas não continham massa de lesão epileptogênica. A análise correlacionou o resultado e as medidas combinadas de eletroencefalografia e o estudo volumétrico da formação hipocampal, sugerindo várias utilizações clínicas para a informação volumétrica, como por exemplo, ajudar na seleção de pacientes para cirurgia, alertar os pacientes das possíveis consequências pós-operatória, e selecionar 
pacientes individuais para o monitoramento pré-operatório invasivo. Segundo o autor, quando a patologia é unilateral, ou seja, a variação de volume e o sinal de Eletroencefalografia são concordantes, sugere-se que os pacientes possam ser operados sem o uso de monitoramento pré-operativo invasivo. Por outro lado, quando o resultado do estudo do volume da formação do hipocampo não é lateralizado, a probabilidade de resultado satisfatório é consideravelmente baixa (42\%), e sempre mais baixa ainda se os dois estudos não tiveram concordância (33\%), sugerindo-se, assim, que o monitoramento invasivo deva ser aplicado ([JACK JR.-1992b]).

\subsection{Mal de Alzheimer}

Embora a precisão do diagnóstico clínico de doenças como o mal de Alzheimer seja razoavelmente alto $(70 \%$ a $90 \%)$, ainda permanecem dificuldades na diferenciação precoce do mal de Alzheimer em relação às mudanças cognitivas encontradas em um envelhecimento normal. [JACK JR.-1992a] propôs o uso de uma técnica baseada em imagens de ressonância magnética para medidas volumétricas do lobo temporal anterior e formação do hipocampo para ajudar no diagnóstico clínico do mal de Alzheimer. O raciocínio para o uso desta técnica no diagnóstico do mal de Alzheimer é o seguinte: (1) o estudo da imagem cerebral é recomendado como parte da avaliação dos pacientes com demência; (2) ressonância magnética é superior a tomografia computadorizada na precisão da imagem do lobo temporal e estruturas límbicas envolvidas na memória.; (3) uma diminuição da memória é frequente no início 
e mais severa na manifestação clínica em pacientes com mal de Alzheimer; (4) estruturas límbicas do lobo temporal, em particular a formação hipocampal, estão intimamente envolvidos no aspecto da função da memória que foi perdida no mal de Alzheimer; e (5) estas estruturas do sistema límbico são extensivelmente envolvidas histopatologicamente no mal de Alzheimer.

Artigos recentes tem sugerido que a medida volumétrica da formação hipocampal sozinha não é suficiente, e que a avaliação de outras estruturas do lobo temporal mesial são necessárias para diferenciar pacientes com mal de Alzheimer suave de indivíduos com envelhecimento normal, pois ainda não está claro qual região do lobo temporal mesial degenera-se primeiro no início do mal de Alzheimer ([MIZUNO2000]).

O estudo volumétrico publicado por [MIZUNO-2000] concluiu que os volumes das estruturas do lobo temporal mesial decaíram com o avanço da severidade do mal de Alzheimer. Em especial, o estudo demonstrou uma diminuição significativa no volume da amígdala no mal de Alzheimer muito sutil, mas não no hipocampo anterior ou posterior. Há controvérsias sobre quais das estruturas do lobo temporal mesial são mais afetadas no estágio inicial do mal de Alzheimer. Acredita-se que o hipocampo degenera-se predominantemente no estágio inicial, entretanto, alguns estudos recentes tem fornecido evidências que a amígdala começa a atrofiar-se no mal de Alzheimer mais cedo do que acreditava-se antes. Os estudos mostram que todas as estruturas do lobo temporal mesial não se atrofiam simultaneamente. 


\subsection{Volumetria}

O desenvolvimento de técnicas de neuroimagem de alta resolução, tais como as medidas volumétricas da amígdala e hipocampo em ressonância magnética nuclear, tem sido útil na obtenção de informações neuroanatômicas in vivo em várias situações clínicas, como epilepsia do lobo temporal, síndromes amnésticas e mal de Alzheimer. Na epilepsia do lobo temporal, os estudos têm demonstrado que volumes hipocampais significativamente reduzidos confirmam os estudos neurofisiológicos e permitem a lateralização da região epileptogênica, sendo correlacionados com anormalidades neuropatológicas e neuropsicológicas, bem como com o prognóstico após lobectomia temporal. Com o acúmulo de experiência e informação, espera-se que as técnicas quantitativas de Ressonância Magnética Nuclear, assim como outros estudos de neuroimagem, reduzam o número de pacientes que necessitam de monitorização eletroencefalográfica invasiva, prolongada e cara, permitindo que mais pacientes sejam tratados de forma adequada utilizando monitorização Eletroencefalografia não invasiva, associada a técnicas de imagem não invasivas e estudos neuropsicológicos ([WHITWELL-2001]).

Ressonância Magnética Nuclear quantitativa permite também a correlação de volumes hipocampais e do complexo amigdalóide pré e pós-operatórios com achados clínicos, neuropsicológicos e neuropatológicos. A adição das medidas do volume amigdalóide às do hipocampo pode permitir a verificação da contribuição de cada uma destas estruturas na epileptogênese e na função de memória. A quantificação pós-operatória da extensão de ressecção amigdalóide e hipocampal pode permitir a 
melhor compreensão de quais estruturas necessitam ser removidas e qual volume de ressecção será necessária. Dados preliminares sugerem que as medidas dos volumes amigdalóide e hipocampal podem também fornecer informações adicionais em pacientes com crises temporais bilaterais, quando usadas em associação com Eletroencefalografia, estudos neuropsicológicos e estudos de neuroimagem funcional.

Para se obter resultados precisos e reproduzíveis com medidas volumétricas do hipocampo e amígdala em Ressonância Magnética Nuclear, vários aspectos técnicos devem ser verificados. O primeiro diz respeito à aquisição e ao processamento da imagem. Resumidamente, o procedimento envolvido nas medidas volumétricas do hipocampo e amígdala em Ressonância Magnética Nuclear necessita de imagens coronais contíguas, finas (1-3 mm), obtidas perpendicularmente ao longo do eixo do hipocampo em aparelhos de alta resolução. As imagens são então transferidas para uma estação de trabalho computadorizada, e os contornos da amígdala e hipocampo são delineados manualmente, seqüencialmente em cada corte, do sentido anterior para posterior. O computador calcula os volumes contando o número de voxels presentes em função da região de interesse a ser medida. O número de voxels é então multiplicado pelo volume de cada voxel para dar o volume total de cada estrutura em $\mathrm{mm}^{3}$ ([WATSON-1998]).

Várias fontes de erro podem ser introduzidas na medida da variação do volume derivado de imagens seriais de um indivíduo. Estas incluem diferenças na quantidade de artefatos de movimento, alterações no campo magnético e variações no tamanho do voxel devido à variação na calibração do aparelho. O trabalho de [WHITWELL-2001] confirmou que inconsistências significantes no tamanho do voxel existem entre imagens seriais, mesmo em máquinas modernas, com mudanças de voxel 
produzindo um artefato aparente de "esticamento" do cérebro. Estes erros tendem a ser mais relevantes que outras fontes de artefatos ([WHITWELL-2001]).

Para serem obtidos resultados precisos e confiáveis com medidas volumétricas por Ressonância Magnética Nuclear, a pessoa que mede os volumes deve ter conhecimento detalhado da anatomia da região temporal medial. Além disso, as estruturas devem ser medidas consistentemente de acordo com um protocolo prédeterminado e padronizado. Quando os limites do hipocampo e amígdala são medidos por um médico especialista, seguindo um protocolo pré-determinado e padronizado, a exatidão e reprodutibilidade das medidas são muito altas ([WATSON-1998]).

Segundo [INSAUSTI-1998] é geralmente aceito que a variabilidade interindividual do tamanho da cabeça afeta os volumes de várias regiões do cérebro, incluindo a amídala e o hipocampo. Para investigar se os volumes dos córtex entorrinal, perirrinal e temperopolar variam com o tamanho da cabeça, os autores correlacionaram os volumes para cada região cortical com a medida da área intracranial no nível da comissura anterior dos mesmos sujeitos, encontrando um relacionamento linear entre o volume do córtex temporopolar e a área intracranial. Os volumes absolutos, assim obtidos, são então normalizados e corrigidos para variações individuais do tamanho do crânio, usando relações pacientes-controle para o volume cerebral total ou volume intracraniano total. Este procedimento aumenta a sensibilidade dos valores absolutos por reduzir os desvios-padrão dos valores do grupo-controle normal. Ele também permite a comparação direta dos valores obtidos de indivíduos com cabeças "grandes" e "pequenas" (Tabela 2.1) ([WATSON-1998]). Segundo [WHITWELL-2001], isso também pode ser utilizado para controle de variações no tamanho do voxel. Esta hipótese assume que 1) o volume total intracranial é inalterado pelo processo da doença, 
e 2) volumes total ou regional são mais comparáveis quando normalizados pelo Volume total intracranial. Em doenças degenerativas, onde o total do cérebro está sujeito a atrofia, o Volume total intracranial pode propiciar a melhor estimativa disponível do volume cerebral antes da doença.

Obtenha a média "Volume Intracraniano Total (VIT)" do grupo-controle normal "Normalize" o volume da FH (ou AM) = R x Volume da FH (ou AM)

Onde, $\mathrm{R}=$ média VIT dos controles VIT dos pacientes

Tabela 2.1. Normalização das medidas volumétricas da formação hipocampal (FH) e amígdala (AM) em Ressonância Magnética Nuclear

Geralmente, imagens em T2 tem sido utilizadas para medir o volume total intracranial porque a alta intensidade de sinal de CSF (Cerebrospinhal Fluid) torna a identificação da superfície do Volume total intracranial relativamente fácil ([WHITWELL-2001]). Vários pesquisadores calculam a relação comparando os tamanhos das amígdalas esquerda e direita, e hipocampos esquerdo e direito. Segundo os estudos de [WATSON-1998], simplesmente divide-se o volume da estrutura menor pelo da maior para obter uma medida da simetria entre os dois lados. Os volumes absolutos das duas amígdalas e dos dois hipocampos são também obtidos, e estes valores absolutos e suas relações comparados com os de uma população-controle normal. Na interpretação dos dados, o diagnóstico de atrofia amigdalóide e hipocampal é feito quando os volumes absolutos são pelo menos dois desvios-padrão menores do que os da população-controle ou se as relações são menores do que 0,90 . Para 
([WHITWELL-2001]), alguns estudos tem utilizado estimativas simples de medidas que correlacionam com o volume total intracranial (por exemplo, espessura intracranial ou a área da seção transversal), mas estas medidas não podem ser consideradas satisfatórias por causa da grande diferença da forma da cabeça dos indivíduos e porque medidas de reprodutibilidade dependem da escolha da posição e o plano de visão.

Além de validar a precisão e reprodutibilidade das medidas de volume, cada centro deve também estabelecer os valores normais presentes em sua população de pacientes e controles. Numerosos fatores entram nos valores absolutos de cada instituição, portanto esperam-se discrepâncias entre as várias instituições, o que requer que cada uma crie seu banco de dados normal ([WATSON-1998]).

A concordância entre as análises visual e volumétrica confirma a confiabilidade da análise visual qualitativa, como indicador da lateralização da área epileptogênica, na suspeita clínica de esclerose mesial temporal, e indica a utilidade da volumetria hipocampal como uma medida suplementar, objetiva e quantitativa para avaliação da esclerose mesial temporal. ([ROGACHESKI-1998])

A discriminação visual entre um hipocampo normal e anormal é direta quando um é evidentemente normal e o outro grosseiramente anormal, porém a comparação visual não funciona em casos de doenças com perdas volumétricas simétricas. Para se determinar de forma precisa a presença e severidade da atrofia nos dois hipocampos, é necessária uma avaliação quantitativa absoluta. Resultados de estudos preliminares indicam que a presença e severidade da esclerose nos dois hipocampos pode fornecer informações prognósticas úteis sobre o controle das crises pós-operatórias e evolução da memória ([WATSON-1997]) . Atualmente, um prognóstico exato não é possível, sendo a determinação precisa dessa relação uma área 
de pesquisa importante na qual a avaliação volumétrica a partir de imagens de ressonância magnética se configura como técnica ideal de investigação.

Lemieux et al. publicou um estudo onde utiliza dois métodos para definir regiões de interesse em fatias individuais: traçado manual e crescimento de região. No traçado manual, o volume da região de interesse é calculado pela multiplicação do volume do voxel $\left(\mathrm{mm}^{3}\right)$ pelo número de pixels da tela contido dentro do traçado, dividido pelo quadrado do fator de magnificação e, no método de crescimento de região, é contado o número de voxels conectados nos dados da imagem original, sendo independente da magnificação. Os volumes de cada fatia individual são somados para obter-se o volume total da estrutura ([LEMIEUX-2000]).

MIZUNO-2000 apresenta um trabalho no qual todas as medidas do volume foram obtidas através de uma técnica de segmentação combinada com traçado e "thresholding". As bordas da formação do hipocampo, a amigdala e o córtex parahipocampal foram manualmente traçadas com o mouse, da imagem posterior para a anterior. Depois que as fronteiras das estruturas anatômicas do lobo temporal mesial foram identificadas, o número de pixels em cada uma foi automaticamente contado e multiplicado pelo tamanho do voxel, para obter um valor numérico em milímetros cúbicos. Os valores das estruturas do lobo temporal mesial individual foram normalizadas para o tamanho da cabeça pela divisão de cada valor pela medida do volume total intracranial ([MIZUNO-2000]). 


\subsection{Scion Image Explorer}

Scion Image é um programa de processamento e análise de imagem para IBM PC. É baseado no popular NIH Image da plataforma Macintosh ([www.scioncorp.com]). Através dele pode-se adquirir, mostrar, editar, realçar, analisar e animar imagens, ler e gravar arquivos TIFF e BMP, propiciando compatibilidade com muitas outras aplicações, incluindo programas para digitalização, processamento, edição e análise de imagens. Suporta muitas funções padrão de processamento de imagens, incluindo realce de contraste, "density profiling", "smoothing", "sharpening", detecção de bordas, filtro mediano e convolução espacial. Pode ser utilizado para medida de área, "mean", "centroid" e perímetro de regiões definidas pelo usuário, mas não efetua o cálculo do volume.

Pode-se editar imagens com escala de cor ou de cinza, incluindo o desenho de linhas, retângulos e textos. Pode-se girar, rotacionar, inverter e escalar seleções. Suporta múltiplas janelas e 8 níveis de magnificação.

A análise do funcionamento do Scion Image foi fundamental como base para a estruturação do projeto, uma vez que era o programa utilizado pelos médicos especialistas e possui quase todas as funções necessárias. A desvantagem deste programa é seu custo consideravelmente alto e não efetuar o cálculo do volume das estruturas selecionadas, sendo necessário para tal a incorporação de planilhas eletrônicas. As figuras 2.2 e 2.3 apresentam exemplos de telas do Scion Image. 


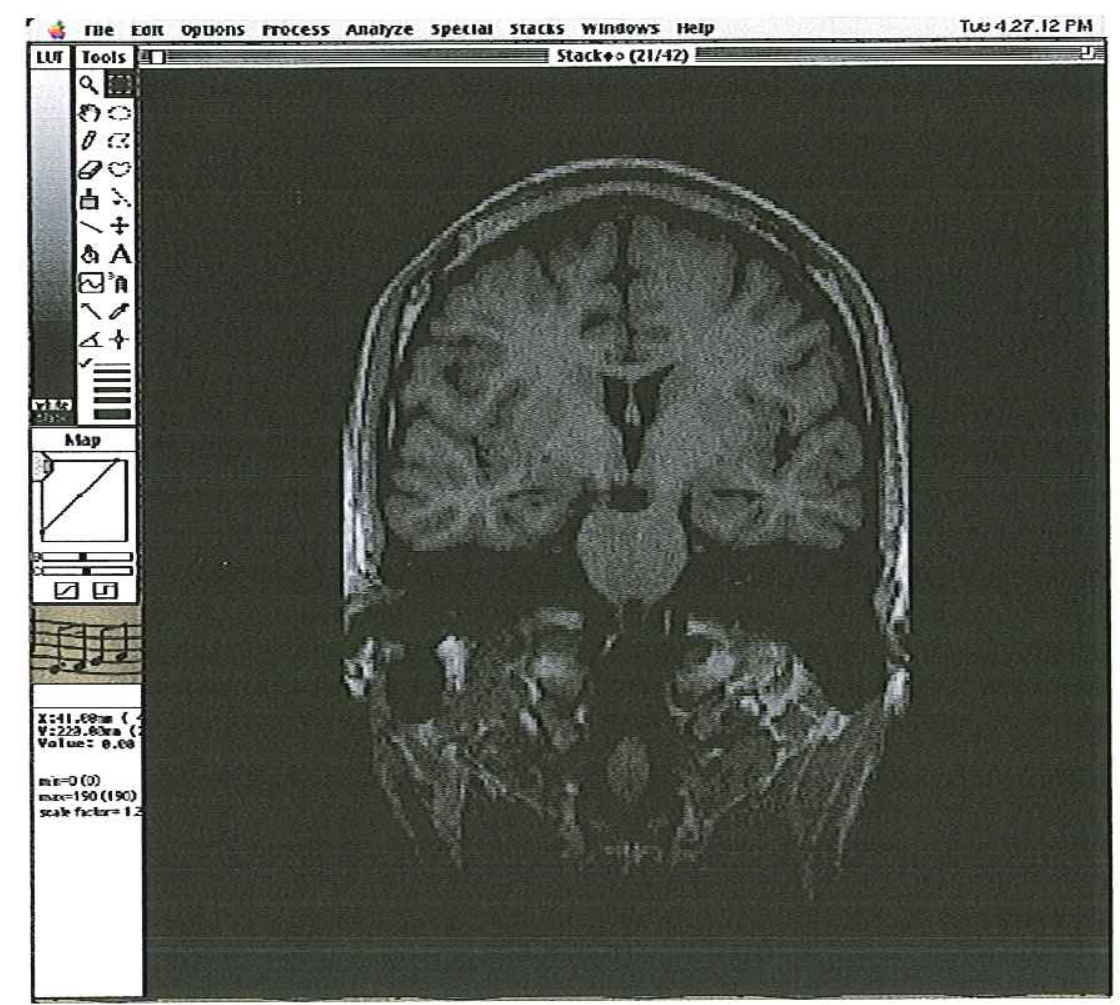

Figura 2.2 - Scion Image em plataforma Macintosh 


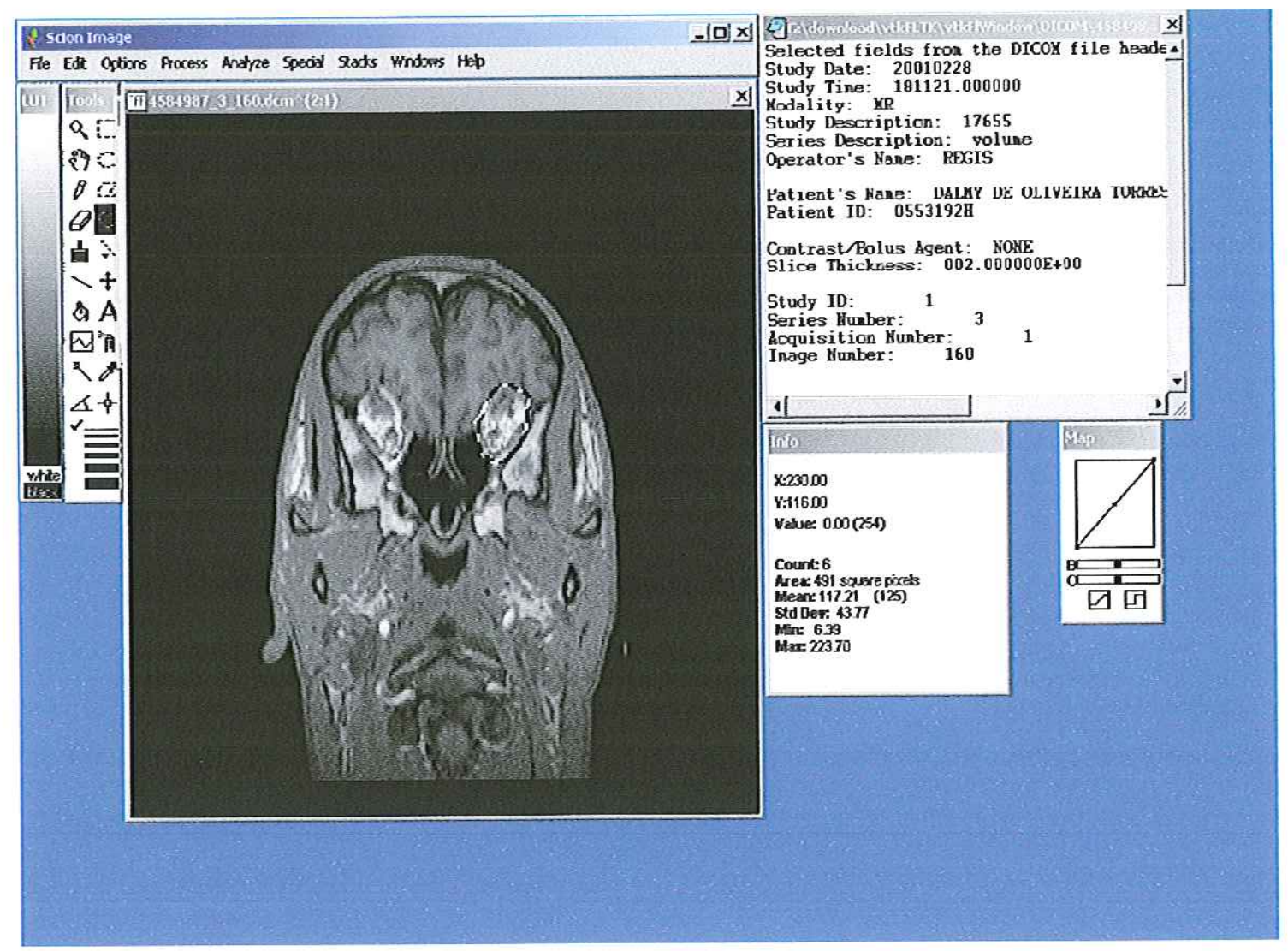

Figura 2.3 - Scion Image em plataforma Windows);

Com esta limitação, o procedimento adotado pelos médicos para cada estudo é o seguinte:

- Criar uma planilha eletrônica para este estudo;

- Organizar as imagens.

Para cada imagem selecionada:

- Abrir a imagem; 
- Marcar as áreas das estruturas uma a uma;

- Transferir o valor da área de cada estrutura individualmente para uma célula da planilha eletrônica.

Isso deve ser feito para cada estrutura a ser quantificada na imagem, visto que não há uma maneira de guardar a área selecionada na imagem através do Scion Image, o único valor armazenado é o que o médico transfere para a planilha eletrônica.

Sendo assim, a confiabilidade deste processo depende totalmente do médico especialista que está manuseando o software, o que é uma tarefa exaustiva e com margem de erro potencialmente elevada pois se o médico trocar o valor de uma célula na hora de transferir a informação o resultado final do estudo será totalmente alterado. 
Capitulo 3

Padrão DICOM 3.0

\subsection{Histórico}

Digital Imaging and COmmunications in Medicine (DICOM) é um padrão para comunicação de imagens médicas e informações associadas. Ele pode ser utilizado por diversas modalidades de equipamentos, como, por exemplo, tomografia computadorizada, ressonância magnética, medicina nuclear e ultra-som. ([FREIRE1997], [NEMA-1999], [BIDGOOD-1998], [CARITÁ-2000], [REVET-1997], [SANTOS-2000], [STASIU-2000]).

Este padrão foi desenvolvido por um comitê de trabalho formado por membros do American College of Radiology (ACR) e do National Electrical Manufactures Association (NEMA) que iniciou os trabalhos em 1983. A primeira versão do padrão, chamada de ACR-NEMA 300-1985 (ou ACR-NEMA Version 1.0), foi apresentada em 1985. Em 1988, uma segunda versão, com novos elementos de dados e vários erros e inconsistências da versão anterior corrigidos, foi distribuída com o nome de ACR-NEMA 300-1988 (ou ACR-NEMA Version 2.0). Naquele momento, o comitê de trabalho percebeu que os usuários em potencial do padrão queriam uma interface 
entre seus equipamentos que possibilitasse a comunicação através de redes. A versão 2.0 não possuía as partes necessárias para uma comunicação em rede robusta, pois ela apenas permitia comunicação ponto-a-ponto entre equipamentos de imagens ou com outros tipos de sistemas, e não uma comunicação direta através de uma rede. O comitê percebeu que seriam necessários mais que simples alterações e adições à versão 2.0, sendo necessário refazer toda a estrutura do padrão, mas mantendo ainda compatibilidade com as versões anteriores.([NEMA-1999])

Surgiu então a versão atual, o ACR-NEMA DICOM, também chamado de DICOM 3.0, que foi apresentada em 1993.

\subsection{O Padrão DICOM}

O padrão DICOM baseia-se em um modelo explícito e detalhado dos vários aspectos relevantes para a área de radiologia e imagens médicas (pacientes, imagens, relatórios, etc.), bem como descrições detalhadas destes aspectos e como eles estão relacionados. O modelo geral foi desenhado usando-se diagramas Entidaderelacionamento (ER - Entity-relationship) e tem como objetivo garantir que usuários e fabricantes tenham um entendimento comum da estrutura de dados usada no padrão. $\mathrm{O}$ modelo fornece os elementos de dados abordados pelo padrão e toda a estrutura de relacionamentos e hierarquia destes elementos.

O padrão hoje está essencialmente completo, embora mudanças evolucionárias ainda possam ser feitas. Como um padrão está estável e maduro, sendo 
implementado por uma variedade de companhias de tecnologia DICOM e fabricantes de equipamentos de geração de imagens. ([SIEGEL-1999])

O padrão DICOM facilita a interoperabilidade de equipamentos de imagens médicas pois especifica um conjunto de protocolos a ser seguido pela reivindicação de conformidade dos dispositivos para o padrão, a sintaxe e semântica de comandos e informações associadas as quais podem ser trocadas utilizando-se estes protocolos e a informação que deve ser suprida com uma implementação para que a conformidade do padrão seja reivindicada.

\subsection{Metas do Padrão DICOM}

O padrão DICOM facilita a interoperabilidade de conformidade de reivindicações de dispositivos, em especial:

- Especifica a semântica de comandos e dados associados. Para dispositivos que interagem, devem haver padrões de como os dispositivos são aguardados para a reação de comandos e dados associados, não somente a informação que está sendo movida entre os dispositivos;

- É explícito na definição de requisitos de conformidade de implementações do padrão. Em especial, uma declaração de conformidade deve especificar informação suficiente para determinar as funções para que a interoperabilidade possa ser aguardada com 
outra reivindicação de conformidade do dispositivo;

- Facilita a operação em ambiente de rede, sem o requisito de unidades de interface de rede;

- É construído para acomodar a introdução de novos serviços, facilitando então suporte para aplicações de imagens médicas futuras;

- Faz uso de padrões internacionais existentes. ([NEMA-1999])

A Figura 3.1 demonstra o processo de construção para uma declaração de conformidade, que consiste de três partes:

- Conjunto de objetos de informação que é reconhecido por esta implementação;

- Conjunto de classes de serviço que esta implementação suporta;

- Conjunto de protocolos de comunicação que esta implementação suporta. 


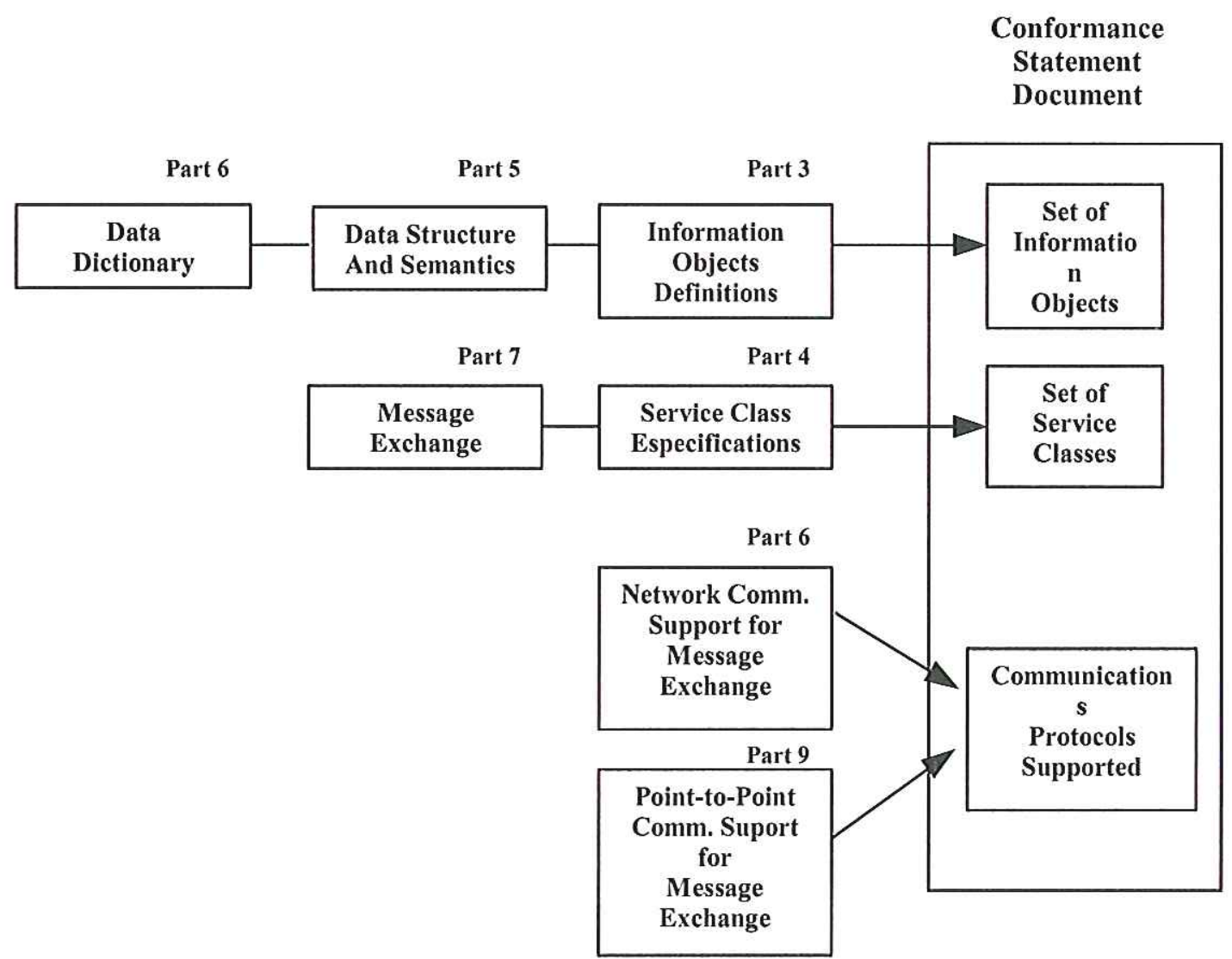

Figura 3.1 - Processo de construção para uma declaração de conformidade.

\subsection{Orientação a Objetos}

O desenvolvimento deste modelo usou uma metodologia de orientação a objetos, onde os objetos são as entidades ou coleção de entidades, definidas no modelo. Para descrever as características de cada objeto existem os atributos. O padrão DICOM chama os objetos que são baseados nos modelos ER (Entity-relationship)do mundo real de objetos de informação (IO - Information Object), e os modelos e tabelas com os 
atributos que definem os IOs de definições dos objetos de informação (IOD Information Object Definition). Se valores reais são substituídos pelos atributos temos então uma entidade chamada de instância. ([NEMA-1999])

A orientação a objetos proporciona não apenas uma maneira de descrever as informações existentes no modelo, mas o que fazer com estas informações ou como obter informações sobre a coleção de objetos existentes. O padrão DICOM faz uso deste conceito para definir serviços, como "armazenar imagem" ou "obter informações do paciente". Estes serviços são implantados no DICOM usando construções chamadas de operações e notificações.

\subsection{Partes do Padrão DICOM}

A versão atual do padrão DICOM 3.0 consiste de nove partes, como mostrado na Figura 3.2. As partes 10 e 11 indicam as próximas extensões ao padrão para suportar a comunicação de arquivos em meios removíveis (discos, fitas, etc). Os módulos do lado direito farão parte de futuras versões do padrão. É importante notar que as partes $1,2,3,5$ e 6 são utilizadas tanto pelos módulos da parte esquerda quanto pelos módulos da parte direita, como sugere a diagramação em camadas da figura. 


\begin{tabular}{|c|c|c|c|c|}
\hline \multicolumn{5}{|l|}{ Part 1: Overview } \\
\hline \multicolumn{5}{|c|}{ Part 2: Conformance } \\
\hline \multicolumn{2}{|c|}{ Part 4: Service Class } & & \multicolumn{2}{|c|}{ Part 11: Media Storage } \\
\hline \multicolumn{2}{|l|}{ Specifications } & $\begin{array}{l}\text { Part 3: } \\
\text { Informe }\end{array}$ & \multicolumn{2}{|c|}{ Application Profiles } \\
\hline \multicolumn{5}{|c|}{ Part 5: Data Structures and Semantics } \\
\hline \multicolumn{5}{|c|}{ Part 6: Data Dictionary } \\
\hline \multicolumn{2}{|c|}{$\begin{array}{l}\text { Part 7: Message Exchange } \\
\text { (Network Operations) }\end{array}$} & \multicolumn{3}{|c|}{ Part 10: Media Storage \& File Format } \\
\hline \multirow{2}{*}{$\begin{array}{l}\text { Part 8: Network } \\
\text { Support }\end{array}$} & \multirow{2}{*}{$\begin{array}{l}\text { Part 9: Point } \\
\text { to Point }\end{array}$} & Part X & Part Y & Part Z \\
\hline & & & $\begin{array}{c}\text { ic Media } \\
\& \\
\text { hysical N }\end{array}$ & \\
\hline
\end{tabular}

Figura 3.2 - Partes atuais do padrão DICOM e partes propostas para extensão do padrão.

A versão 3.0 do padrão DICOM é essencialmente voltada para comunicação entre equipamentos, seja através de redes ou por ligações ponto-a-ponto. A idéia é que cada equipamento possa usar os seus próprios padrões e formatos para armazenar e gerenciar os seus dados, mas quando se deseja comunicar com outros equipamentos, torna-se importante a existência de uma linguagem comum que equipamentos de diversos fabricantes sejam capazes de entender. Por outro lado, muitos laboratórios ou centros de diagnóstico não operam em rede e os resultados muitas vezes devem ser mostrados de maneira especial para especialistas externos, como por exemplo, um conjunto de imagens cardíacas apresentadas em forma dinâmica. Para estes casos, surge a necessidade de armazenar as informações em meios físicos removíveis para que ela possa ser transportada. Um primeiro passo para atender esta necessidade foi a criação da 
parte número 10 do padrão, que faz descrições genéricas de estruturas de arquivos e diretórios para meios físicos removíveis. As partes 11 e 12 ainda estão sendo desenvolvidas. A parte 11 irá exercer um papel semelhante à parte 4 do padrão, na designação das classes. Já a parte 12 irá especificar os detalhes de armazenamento em cada tipo de meio físico escolhido para fazer parte deste padrão.

A Tabela 3.1 a seguir resume o conteúdo de cada parte do padrão.

\begin{tabular}{|l|l|}
\hline Parte 1: Overview & $\begin{array}{l}\text { Apresentação do padrão, com uma descrição dos } \\
\text { princípios de desenvolvimento utilizados, definição } \\
\text { da terminologia e descrição das demais partes do } \\
\text { padrão. }\end{array}$ \\
\hline Parte 2: Conformance & $\begin{array}{l}\text { Define os termos de conformidade com o padrão, } \\
\text { indicando como os fabricantes devem descrever sem } \\
\text { ambigüidade como seus produtos estão em } \\
\text { conformidade com o padrão. }\end{array}$ \\
\hline Parte 3: Information Objects & $\begin{array}{l}\text { Descreve como os IOs são definidos e especifica as } \\
\text { diversas classes de IO usadas no padrão. Muitos IODs } \\
\text { possuíam grupos de atributos comuns ou similares, de } \\
\text { forma que estes foram reunidos para criar módulos } \\
\text { comuns que podem ser usados por mais de um IOD. } \\
\text { Assim foram criados IODs compostos e IODs } \\
\text { normalizados. }\end{array}$ \\
\hline $\begin{array}{l}\text { Parte 4: Service Class } \\
\text { Specifications }\end{array}$ & $\begin{array}{l}\text { Contém as especificações das classes de serviço, que } \\
\text { são: } \\
\text { Certification Service Class } \\
\text { Storage Service Class } \\
\text { Query Retrieve Service Class } \\
\text { Study Content Notification Service Class } \\
\text { Patient Management Service Class } \\
\text { Study Management Service Class } \\
\text { Results Management Service Class } \\
\text { Print Management Service Class }\end{array}$ \\
\hline And Semantics & $\begin{array}{l}\text { pefine como um conjunto de informações } \\
\text { provenientes de objetos de informação e de classes de } \\
\text { serviços devem ser codificadas para fazerem parte de } \\
\text { uma mensagem. }\end{array}$ \\
\hline arte
\end{tabular}




\begin{tabular}{|l|l|}
\hline Parte 6: Data Dictionary & $\begin{array}{l}\text { Fornece uma lista de todos elementos de dados, ou } \\
\text { atributos, que compõe todos os IOs. Para cada } \\
\text { elemento de dado é fornecido o seu código numérico, } \\
\text { o seu nome, sua representação (texto, número em } \\
\text { ponto-flutuante, etc.), a multiplicidade e o domínio de } \\
\text { valores permitidos. }\end{array}$ \\
\hline $\begin{array}{l}\text { Parte 7: Message Exchange } \\
\text { (Network Operations) }\end{array}$ & $\begin{array}{l}\text { Aqui é descrita a dinâmica de comunicação, } \\
\text { indicando o que é necessário para uma aplicação } \\
\text { interagir no padrão de comunicação do DICOM. Esta } \\
\text { parte define como são construídas as seqüências de } \\
\text { comandos, da mesma forma que a parte 5 define } \\
\text { como são construídas as seqüências de dados. }\end{array}$ \\
\hline $\begin{array}{l}\text { Parte 8: Network Support } \\
\text { TCP/IP \& OSI }\end{array}$ & $\begin{array}{l}\text { Define o suporte de rede necessário para a troca de } \\
\text { mensagens do DICOM. Atualmente o protocolo } \\
\text { TCP/IP e qualquer outro que satisfaça o modelo de } \\
\text { camadas ISO-OSI são suportados, mas a estrutura do } \\
\text { padrão permite que novos protocolos sejam } \\
\text { incorporados no futuro. }\end{array}$ \\
\hline Parte 9: Point to Point & $\begin{array}{l}\text { Para manter compatibilidade com as versões } \\
\text { anteriores do padrão, que usavam interfaces paralelas } \\
\text { de dados de alta velocidade, o protocolo de } \\
\text { comunicação ponto-a-ponto foi mantido. }\end{array}$ \\
\hline
\end{tabular}

Tabela 3.1 - Resumo das partes do padrão DICOM 3.0 ([FREIRE-1997])

\subsection{Tags}

Uma Tag é um identificador único para um elemento de informação composto de um par ordenado de números (um número do grupo seguido por um número de elemento), o qual é usado para identificar atributos e os elementos de dados correspondentes. ([NEMA-1999]) 
As Tags do grupo 0010, por exemplo, representam as informações dos pacientes, tais como nome, código de identificação, data de aniversário, sexo, idade, entre outros. (Tabela 3.2)

$\begin{array}{ll}\text { Tag } & \text { Name } \\ (0010,0000) & \text { Group Length } \\ (0010,0020) & \text { Patient ID } \\ (0010,0021) & \text { Issuer of Patient ID } \\ (0010,0010) & \text { Patient's Name } \\ (0010,0030) & \text { Patient's Birth Date } \\ (0010,0032) & \text { Patient's Birth Time } \\ (0010,0040) & \text { Patient's Sex } \\ (0010,0050) & \text { Patient's Insurance Plan Code Sequence } \\ (0010,1000) & \text { Other Patient IDs } \\ (0010,1001) & \text { Other Patient Names } \\ (0010,1005) & \text { Patient's Birth Name } \\ (0010,1010) & \text { Patient's Age } \\ (0010,1030) & \text { Patient's Weight } \\ (0010,1020) & \text { Patient's Size } \\ (0010,1040) & \text { Patient's Address } \\ (0010,1060) & \text { Patient's Mother's Birth Name } \\ (0010,1080) & \text { Military Rank } \\ (0010,1081) & \text { Branch of Service } \\ (0010,1090) & \text { Medical Record Locator } \\ (0010,2000) & \text { Medical Alerts } \\ (0010,2110) & \text { Contrast Allergies } \\ (0010,2150) & \text { Country of Residence } \\ (0010,2152) & \text { Region of Residence } \\ (0010,2154) & \text { Patient's Telephone Numbers } \\ (0010,2160) & \text { Ethnic Group } \\ (0010,2180) & \text { Occupation } \\ (0010,21 A 0) & \text { Smoking Status } \\ (0010,21 B 0) & \text { Additional Patient History } \\ (0010,21 C 0) & \text { Pregnancy Status } \\ (0010,2100) & \text { Last Menstrual Date } \\ (0010,21 F 0) & \text { Patient's Religious Preference } \\ (0010,4000) & \text { Patient Comments } \\ & \\ & \\ (00) & \end{array}$

Tabela 3.2 - Tags do grupo 0010 - Informações sobre o Paciente ([NEMA-1999])

A Tabela 3.3 lista algumas tags que foram extraídas de uma imagem

DICOM e que são importantes para a identificação de todas as imagens pertencentes a uma seqüência de exames. 


\begin{tabular}{|ll|}
\hline Tag & Name \\
$(0008,0060)$ & Modality \\
$(0008,0070)$ & Manufacturer \\
$(0008,0080)$ & Institution Name \\
$(0008,1030)$ & Study Description \\
$(0008,103 E)$ & Series Description \\
$(0009,1226)$ & Study date \\
$(0010,0010)$ & Patient's Name \\
$(0010,0020)$ & Patient ID \\
$(0010,0030)$ & Patient's Birth Date \\
$(0010,0040)$ & Patient's Sex \\
$(0010,1010)$ & Patient's Age \\
$(0018,0024)$ & Sequence name \\
$(0018,1250)$ & Receiving Coil \\
$(0020,0013)$ & Instance Number \\
$(0028,0010)$ & Rows \\
$(0028,0011)$ & Columns \\
$(0028,0100)$ & Bits Alocated \\
$(0028,0101)$ & Bits Stored \\
$(0028,0102)$ & High Bit \\
$(7 F E 0,0010)$ & Pixel Data \\
& \\
\hline
\end{tabular}

Tabela 3.3 - Tags reconhecidas e utilizadas para o trabalho.

A Tabela 3.4 exemplifica alguns valores para as Tags mostradas na Tabela 3.3: 


\begin{tabular}{|ll|}
\hline Tag & Value \\
$(0008,0060)$ & MR \\
$(0008,0070)$ & SIEMENS \\
$(0008,0080)$ & HC FMUSP RIB. PRETO \\
$(0008,1030)$ & 13222 \\
$(0008,103 \mathrm{E})$ & encefalo-malfvasc/s cout \\
$(0009,1226)$ & 20020331 \\
$(0010,0010)$ & LUCIENE CAVALCANTI \\
$(0010,0020)$ & $12345678 \mathrm{~B}$ \\
$(0010,0030)$ & 19780602 \\
$(0010,0040)$ & $\mathrm{F}$ \\
$(0010,1010)$ & $023 \mathrm{Y}$ \\
$(0018,0024)$ & scout \\
$(0018,1250)$ & CP Head \\
$(0020,0013)$ & Instance Number \\
$(0028,0010)$ & 256 \\
$(0028,0011)$ & 256 \\
$(0028,0040)$ & RECT \\
$(0028,0100)$ & 16 \\
$(0028,0101)$ & 12 \\
$(0028,0102)$ & 11 \\
$(7 \mathrm{FE} 0,0010)$ & 1 \\
& \\
\hline
\end{tabular}

Tabela 3.4 - Tags reconhecidas e com seus respectivos valores.

\subsection{DICOM e Outros Formatos}

Embora do padrão DICOM permaneça como o escolhido para troca de imagens médicas, seu relacionamento para outros tipos de padrões deve ser observado. Há padrões gráficos desenvolvidos para troca de imagens gráficas e o padrão HL7 desenvolvido para troca de informação textual em informática médica.

Muitos dos padrões gráficos (GIF, TIFF, etc.) foram desenvolvidos somente para troca de dados da imagem, sem referência ao conteúdo ou ao relacionamento do conteúdo para outros objetos de informação.

HL7 é um padrão sancionado pelo American National Standard Institute 
(ANSI) para comunicação de informação médica, originalmente desenvolvido para trocar dados clínicos de pacientes. Este padrão tem sido utilizado para facilitar a troca de informações e imagens entre sistemas baseados no protocolo DICOM, ou seja, utilizar mensagens HL7 para entregar dados DICOM ([OOSERWIJK-1998)].

O HL7 está concentrado no nível de aplicação padronizando o modelo de dados. Com a utilização do HL7 é possível definir os dados e as mensagens a serem transferidos pela rede. Corresponde à definição de uma interface de aplicação que especifica os dados a serem trocados, o momento da troca e o tratamento de determinados erros específicos entre as aplicações ([ABRAHÃO-2000]).

O HL7 não procura assumir uma arquitetura particular física ou lógica para a implantação de sistemas de saúde. Ele estabelece um padrão de comunicação entre sistemas diferentes e incompatíveis. O HL7 é um conjunto de transações baseadas em mensagens entre aplicações na área da saúde, que definem regras de codificação, produzem mensagens ASCII delimitadas e de tamanho variável. O protocolo suporta tanto mensagens não solicitadas, que são as mensagens de admissão, alta e transferência de pacientes, como as solicitadas, que são as consultas ([ABRAHÃO-2000]). 
Capítulo 4

Materiais e Métodos

\subsection{Sistema Operacional}

Os algoritmos envolvidos com o processamento de imagens tem um custo computacional bastante alto, necessitando não só de um equipamento robusto como também de um sistema operacional que garanta uma boa performance.

No trabalho são utilizadas imagens obtidas do aparelho de Ressonância Magnética Nuclear, que são anatômicas arquivadas segundo o padrão de imagens médicas DICOM. Devido ao volume elevado de dados é necessário um ambiente operacional estável e de bom desempenho.

A utilização do Sistema Operacional Windows 98/2000 (Microsoft), que é bastante difundido mundialmente e que apresenta várias ferramentas para o desenvolvimento foi descartada pois possui algumas limitações, tais como: limitação na profundidade de pixels visualizados e armazenados, é lento no processamento das imagens, dificuldade de manipulação dos diversos arquivos em um só projeto e problemas de instabilidade. 
A escolha do Linux baseou-se em características já comprovadas e testadas de performance e estabilidade, tais como:

- É gratuito, isto é, o Linux é um clone do sistema operacional (OS) UNIX distribuído gratuitamente.

- É executado na arquitetura barata do PC Intel 386/486/Pentium e suporta uma grande variedade de placas de vídeo, placas de áudio, drives de CD-ROM, drives de discos e outros dispositivos. O Linux pode ser usado nos sistemas EISA, Localbus, ou PCI também. O Linux foi transportado para outras plataformas, incluindo os processadores Motorola, 680x0, DEC ALPHA, PowerPC e SPARC. Outras arquiteturas são também objetivos, e projetos de desenvolvimento estão em andamento.

- O sistema é rápido, mesmo com muitos processos sendo executados e com diversas janelas abertas, pois o Linux faz um excelente uso do hardware. Muitos sistemas operacionais comerciais, como o MSDOS, fazem pouco uso da capacidade avançada com diversas tarefas do processador $80 \times 86$. O Linux é próprio para essa arquitetura e a utiliza de forma completa.

- Tem boa performance e executa as aplicações de alta qualidade. $\mathrm{O}$ Linux está sendo desenvolvido publicamente com centenas de programadores e usuários aperfeiçoando-o. Diferente de outros sistemas operacionais novos, o Linux já tem uma enorme base de aplicações disponíveis gratuitamente para serem utilizadas, desde aplicações científicas maiores até as ferramentas multimídia e jogos. 
- É altamente compatível com MS-DOS e Windows 98. Você poderá instalar o Linux em outras partições do disco que contenham o MSDOS ou outros sistemas operacionais. O Linux pode acessar diretamente os arquivos do MS-DOS a partir de um dispositivo de disquete ou do disco rígido.

\subsection{Linguagem para Desenvolvimento}

O desenvolvimento de um software de confiabilidade e com bom desempenho depende bastante da linguagem que será utilizada para tal. Nesse projeto buscou-se uma linguagem multiplataforma, isto é, uma linguagem que possa ser compatível tanto com os recursos do Linux quanto com outros sistemas operacionais (Windows, OS/2, UNIX, por exemplo), isso devido a pouca quantidade de equipamentos que possuem o sistema operacional Linux e à inegável prevalência de equipamentos que possuem o sistema operacional Windows, que ainda é bastante difundido.

Foi necessária uma linguagem onde a interface gráfica fosse rápida de montar e bastante amigável com o usuário, e sobretudo que fosse veloz no processamento de imagens, devido ao alto custo computacional de processamento e para obtenção de resultados no menor tempo possível.

A linguagem $\mathrm{C} / \mathrm{C}++$ foi escolhida como a que se adequa mais a esses requisitos, porém tem a desvantagem de não ter uma interface gráfica muito amigável e 
prática. Com essa dificuldade para a montagem do ambiente, foi procurada uma solução optando-se pelo VTK (Visualizaton ToolKit), por se adaptar melhor aos requisitos citados em conjunto com o FLTK (Fast Light Tool Kit).

O VTK é um software baseado em orientação a objetos, e muito utilizado atualmente na linha de pesquisa para construção, renderização, e reconstrução de volumes, sendo compatível com Windows e Linux. O VTK é interpretado e pode ser construído por diversas linguagens entre elas C++, OpenGL, TCL e Java, sendo também utilizado para a criação de mundos virtuais utilizando a técnica de VRML. Consiste de dois subsistemas básicos: uma biblioteca da classe do compilador $\mathrm{C}++$, e um interpretador de camada envoltória que permite manipular classes de compiladores usando as linguagens Java , TCL e Python. A vantagem desta arquitetura é poder construir algoritmos eficientes na linguagem do compilador $\mathrm{C}++$, e reter as características de desenvolvimento do código rápido de linguagens interpretadas, podendo-se construir aplicações diretamente em C++ [SCHROEDER-2000].

O FLTK (Fast Light Tool Kit) é uma ferramenta para criação de interfaces gráficas compatível com Linux (UNIX), OpenGL e Microsoft Windows, sendo atualmente mantido por um pequeno grupo de desenvolvedores em todo o mundo e é um software de domínio público [SWEET-2000]. 


\subsection{Software Desenvolvido}

O software foi desenvolvido utilizando-se a linguagem $\mathrm{C}++$ em conjunto com o VTK para auxiliar na visualização e manipulação de imagens e FLTK para criação da interface gráfica. O sistema operacional utilizado foi o Linux, por ser comprovadamente mais rápido e eficaz para processamento de imagens.

A técnica que foi implementada é a de traçado manual das estruturas de interesse. Com essa técnica o médico especialista traça o contorno das diversas estruturas através do mouse, o software captura os pontos e desenha o contorno fechado da estrutura.

O software processa a imagem do tipo DICOM, reconhece as TAGs, exibe a imagem e disponibiliza a interface gráfica para que o médico especialista possa iniciar a marcação das regiões de interesse para posterior cálculo volumétrico.

Ao iniciar, o software exibe uma interface na qual o usuário é orientado a utilizar um browse que visualiza todo o conteúdo do disco rígido e escolher o diretório onde encontram-se todas as imagens do estudo.

Nesta mesma janela o usuário tem a opção de abrir um estudo já existente ou iniciar um novo estudo. O nome do estudo deverá ser informado para que o software crie todas as configurações necessárias ou encontre o estudo já gravado.

Baseado nas informações sobre o diretório e o nome do arquivo inicia-se o processo de abertura de todas as imagens e reconhecimento das informações para ordená-las de acordo com sua seqüência de aquisição. Visto que o nome dos arquivos diferenciam-se das informações contidas nos mesmos, a cada imagem lida do diretório 
especificado é retirado o número de seqüência da imagem e o nome do arquivo, colocado-os em um vetor (Algoritmo 1).

\section{INÍCIO}

EXIBIR_JANELA_PRINCIPAL() // Figura 1

LOCALIZAR_DIRETÓRIO(Browser) // Figura 2

ENQUANTO Existem arquivos no diretório FAÇA

ABRIR_DICOM(Nome_arquivo)

Vetor_Imagens[contador] $=$ Nome_arquivo

Vetor_Numero_Imagem[contador] = Sequencia_imagem

contador $+=1$

FIMENQUANTO

ORDENAR_VETOR(Vetor_Imagens, Vetor_Numero_Imagem)

ENQUANTO contador $2<=$ contador FAÇA

SE contador $2=0$ ENTÃO

CRIAR_ARQUIVO(Nome do estudo)

ELSE

GRAVAR_NO_ARQUIVO(Nome do estudo)

FIMSE

FIMENQUANTO

VISUALIZAR(Vetor_Imagens[0]) // Figura 3

FIM

Algoritmo 1. Algoritmo para organização, abertura e visualização das imagens. 
Após a leitura de todas as imagens o vetor é ordenado, as informações sobre o estudo e a ordenação das imagens são gravadas em um arquivo, podendo-se assim iniciar a interface gráfica para visualização da primeira imagem a ser marcada.

As estruturas marcadas são diferenciadas por cores, e, ao selecionar-se a ferramenta apropriada o software automaticamente disponibiliza a função para o traçado manual da estrutura escolhida através da interação do médico especialista com o mouse.

O médico especialista deve selecionar a estrutura que deseja marcar na barra de ferramentas, posicionar o ponteiro do mouse sobre o ponto inicial e permanecer com o botão esquerdo do mouse pressionado até que o último ponto da estrutura seja marcado. Ao soltar o botão esquerdo do mouse o software efetuará a união do último ponto marcado com o primeiro, para exibir um contorno fechado da estrutura.

Através deste processo o software efetua a captura de todos os pontos do contorno de cada estrutura, que são armazenados em uma matriz juntamente com a identificação do número da imagem e da estrutura que está sendo marcada em cada corte, servindo como base para calcular o volume.

Cada estrutura que já tenha sido marcada terá seu botão na barra de ferramentas com a sua cor específica, sendo que se o médico especialista tiver a necessidade de redesenhá-la terá que selecionar o botão desejado para retirar a imagem e, então, habilitar a ferramenta para poder efetuar a marcação do contorno de cada estrutura novamente.

Após as estruturas serem marcadas em todos os planos o programa calcula os volumes e apresenta uma tabela de resultados indicando cada estrutura e seu respectivo volume acumulado. O cálculo é feito baseado no princípio de Cavalieri (Algoritmo 2). 


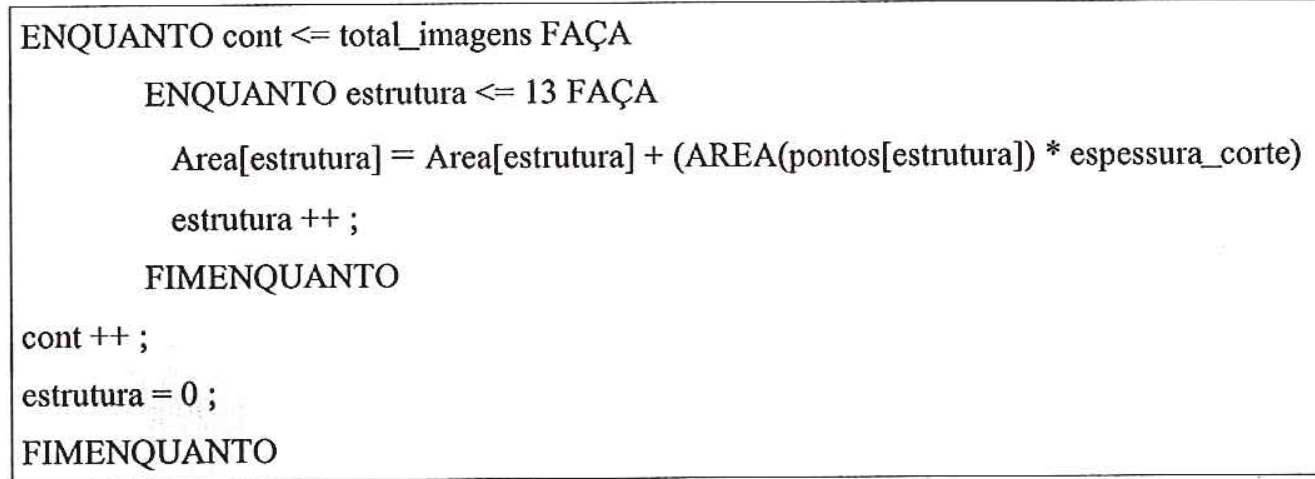

Algoritmo 2. Cálculo do volume de acordo com o princípio de Cavalieri.

Além do volume absoluto das estruturas, ao término do estudo são calculados também os valores normalizados e a assimetria, segundo a tabela 4.1 e exibidos para o usuário segundo a tabela 4.2.

\begin{tabular}{|c|c|}
\hline Assimetria & Valores Normalizados \\
\hline$\left|\left(\begin{array}{c}\text { Direita }- \text { Esquerda } \\
\left(\frac{\text { Direita }+ \text { Esquerda }}{2}\right)\end{array}\right)\right| * 100$ & ||$\left(\frac{\text { Volume da estrutura }}{\text { Volume supra tentorial }}\right) \mid * 100$ \\
\hline
\end{tabular}

Tabela 4.1 - Base de cálculo para a assimetria e os valores normalizados. 


\begin{tabular}{|c|c|c|}
\hline Valores Absolutos & Assimetria & Valores Normalizados \\
\hline $\begin{array}{l}\text { SUPRA TENTORIAL } \\
\text { HIPOCAMPO DIREITO } \\
\text { HIPOCAMPO ESQ. } \\
\text { LOBO DIREITO } \\
\text { LOBO ESQUERDO } \\
\text { AMIGDALA DIREITA } \\
\text { AMIGDALA ESQ. } \\
\text { CORTEX DIREITO } \\
\text { CORTEX ESQ. } \\
\text { CORNO DIREITO } \\
\text { CORNO ESQUERDO } \\
\text { POLO DIREITO } \\
\text { POLO ESQUERDO }\end{array}$ & $\begin{array}{l}\text { HIPOCAMPO } \\
\text { LOBO TEMPORAL } \\
\text { AMIGDALA } \\
\text { CORTEX ENTOHINAL } \\
\text { CORNO TEMPORAL } \\
\text { POLO TEMPORAL }\end{array}$ & $\begin{array}{l}\text { HIPOCAMPO DIREITO } \\
\text { HIPOCAMPO ESQ. } \\
\text { LOBO DIREITO } \\
\text { LOBO ESQUERDO } \\
\text { AMIGDALA DIREITA } \\
\text { AMIGDALA ESQ. } \\
\text { CORTEX DIREITO } \\
\text { CORTEX ESQ. } \\
\text { CORNO DIREITO } \\
\text { CORNO ESQUERDO } \\
\text { POLO DIREITO } \\
\text { POLO ESQUERDO }\end{array}$ \\
\hline
\end{tabular}

Tabela 4.2 - Resultados mostrados ao usuário após o término do estudo.

\subsection{Metodologia para Avaliação do Software}

A calibração do software é feita automaticamente com as informações extraídas do cabeçalho do arquivo DICOM ou a partir de informações inseridas manualmente no início do estudo, caso alguma imagem não possua a informação correspondente.

Para validação do software, um médico especialista efetuou as marcações das estruturas com o Scion Image Explorer e utilizando a ferramenta desenvolvida, obtendo-se assim o grau de correlação entre estes. Existe uma variação intra e interpessoal, visto que não é possível garantir que o médico especialista irá marcar exatamente a mesma quantidade de pontos que marcou anteriormente, além de que existem variações quando dois especialistas diferentes marcam o mesmo estudo, pois a 
experiência profissional conta muito.

Foram comparados 2 casos de pacientes com Mal de Alzheimer e 1 com epilepsia pelo mesmo médico especialista.

A avaliação quantitativa consistiu na comparação dos valores obtidos através das marcações efetuados com o Scion e a ferramenta desenvolvida.

A avaliação qualitativa levou em conta o grau de satisfação do usuário, baseado em aspectos referentes à facilidade de uso, apresentação geral, resultados obtidos, comparativo com o Scion. A resposta dos usuários foi tabelada, considerandose: 1- Ruim, 2 - Regular, 3 - Boa e 4 - Excelente.

Estas avaliações foram efetuadas por dois especialistas da área de neurologia do Hospital das Clínicas da Faculdade de Medicina de Ribeirão Preto. A ferramenta ficou disponível para testes durante 30 dias, dentro dos quais os especialistas utilizam a ferramenta em intervalos de tempo aleatórios para testes com casos cotidianos reais. Em média a ferramenta foi utilizada durante 10 dias, cada médico especialista trabalhou 4 vezes durante um intervalo de tempo de cerca de 30 minutos.

A ferramenta foi submetida a testes utilizando-se comparações com ferramentas de terceiros, imagens já estudadas e segmentação manual de médicos especialistas do Hospital das Clínicas. As patologias avaliadas foram Mal de Alzheimer e Epilepsia de pacientes considerados normais, razoáveis e críticos.

Ao término do estudo são calculados os valores absolutos e normalizados das estruturas: Supra tentorial, Hipocampo Direito (HD), Hipocampo Esquerdo (HE), Lobo Direito (LD), Lobo Esquerdo (LE), Amigdala Direita (AD), Amigdala Esquerda (AE), Córtex Direito (CD), Córtex Esquerdo (CE), Corno Temporal Direito (CTD), 
Corno Temporal Esquerdo (CTE), Polo Temporal Direito (PD) e Polo Temporal Esquerdo (PE), os valores da assimetria bilateral das estruturas: Hipocampo, Lobo Temporal, Amigdala, Córtex Entohinal, Corno Temporal e Polo Temporal, de acordo com as seguintes fórmulas mostradas na Tabela 5.2:

\begin{tabular}{|l|c|c|}
\hline \multicolumn{1}{|c|}{ Valores Absolutos } & Assimetria & Valores Normalizados \\
\hline $\begin{array}{l}\text { Área da estrutura * } \\
\text { espessura do corte }\end{array}$ & ||$\left(\frac{\text { Direita }- \text { Esquerda }}{\left(\frac{\text { Direita }+ \text { Esquerda }}{2}\right)}\right) \| * 100$ & $\left|\left(\frac{\text { Volume da estrutura }}{\text { Volume supra tentorial }}\right)\right| * 100$ \\
\hline
\end{tabular}

Tabela 4.3 - Valores calculados pelo sistema a partir das marcações das estruturas, em $\mathrm{mm}^{3}$. 
Capítulo 5

Resultados Obtidos

\subsection{Interfaces do sistema}

$\mathrm{Na}$ interface principal apresentada ao médico especialista, encontram-se várias ferramentas, tais como manipulação do brilho e contraste, marcação das estruturas de interesse e de navegação entre as imagens. (Figuras 5.1 à 5.4)

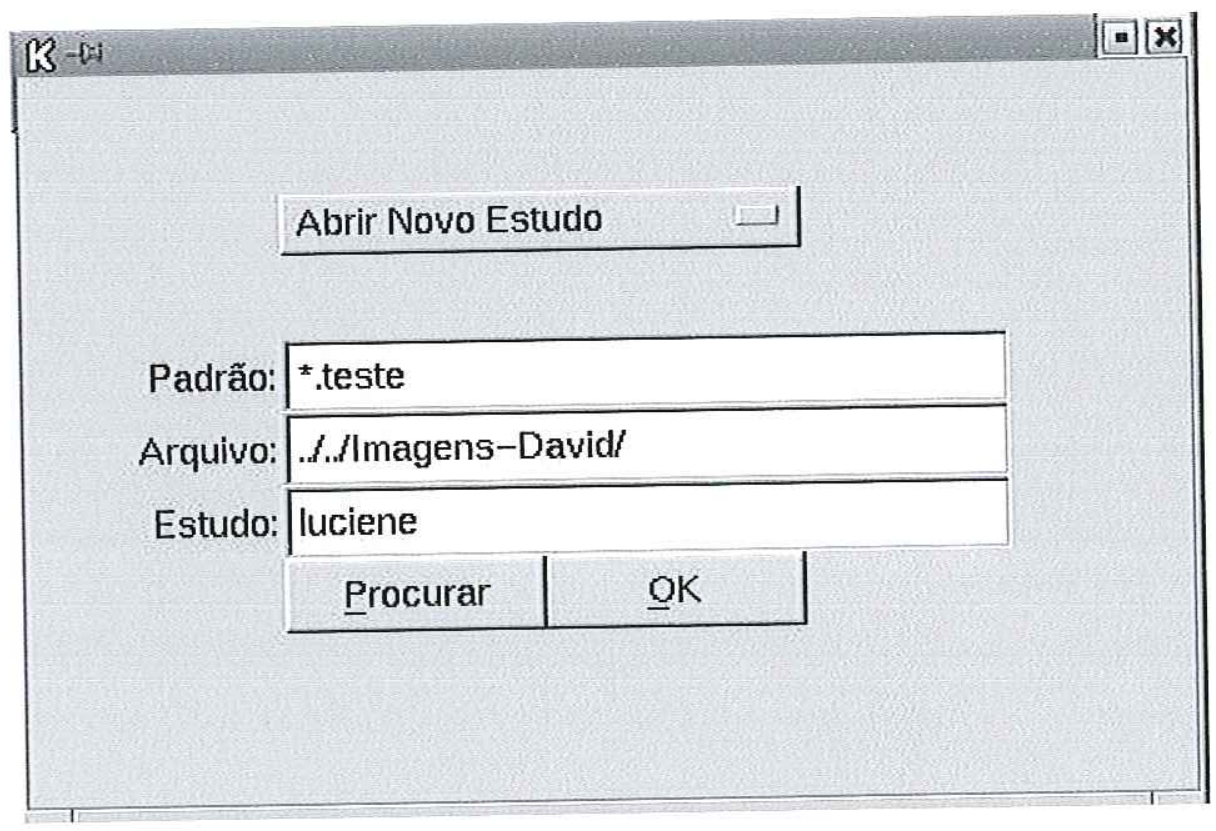

Figura 5.1. Janela Principal do software. 


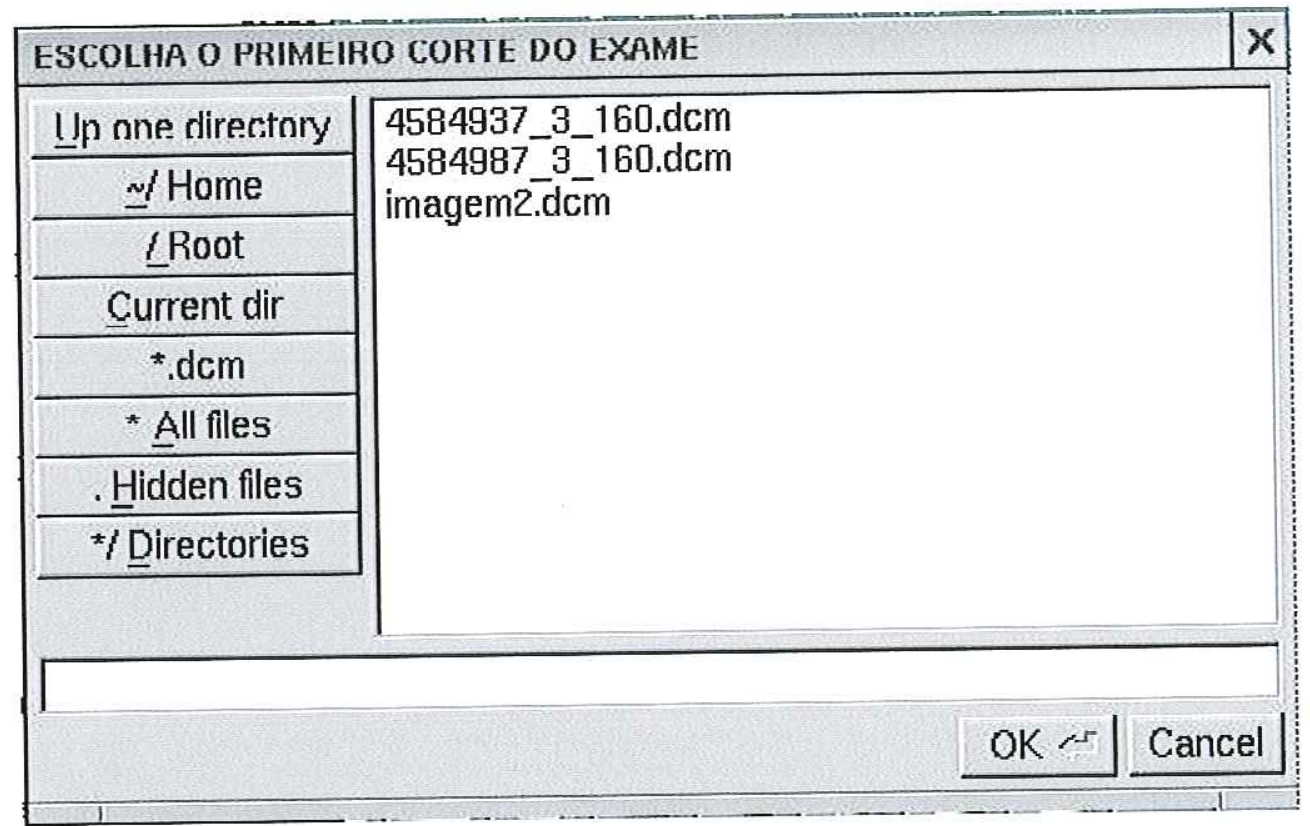

Figura 5.2. Interface para escolha do diretório.

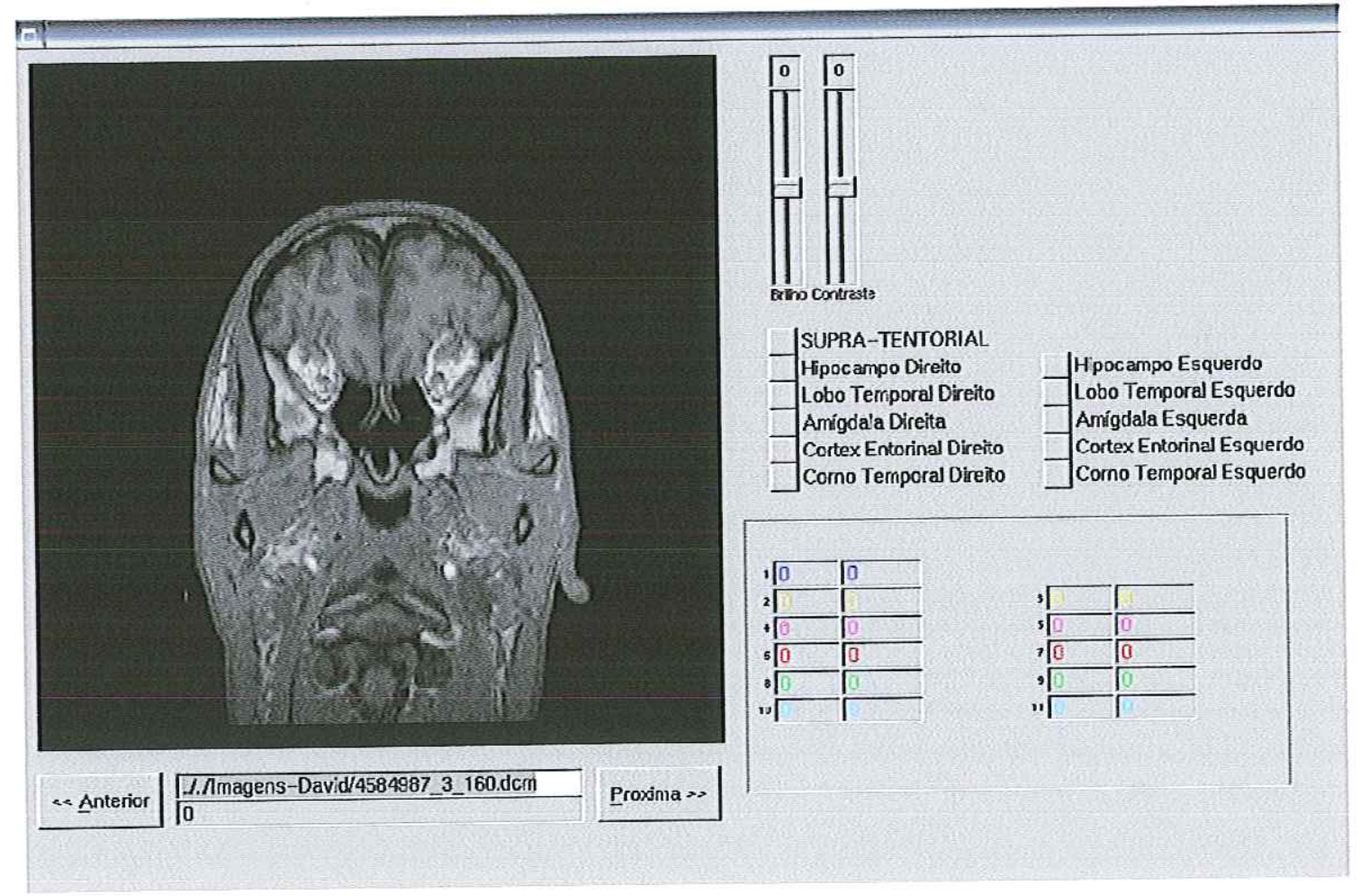

Figura 5.3. Interface com a primeira imagem. 

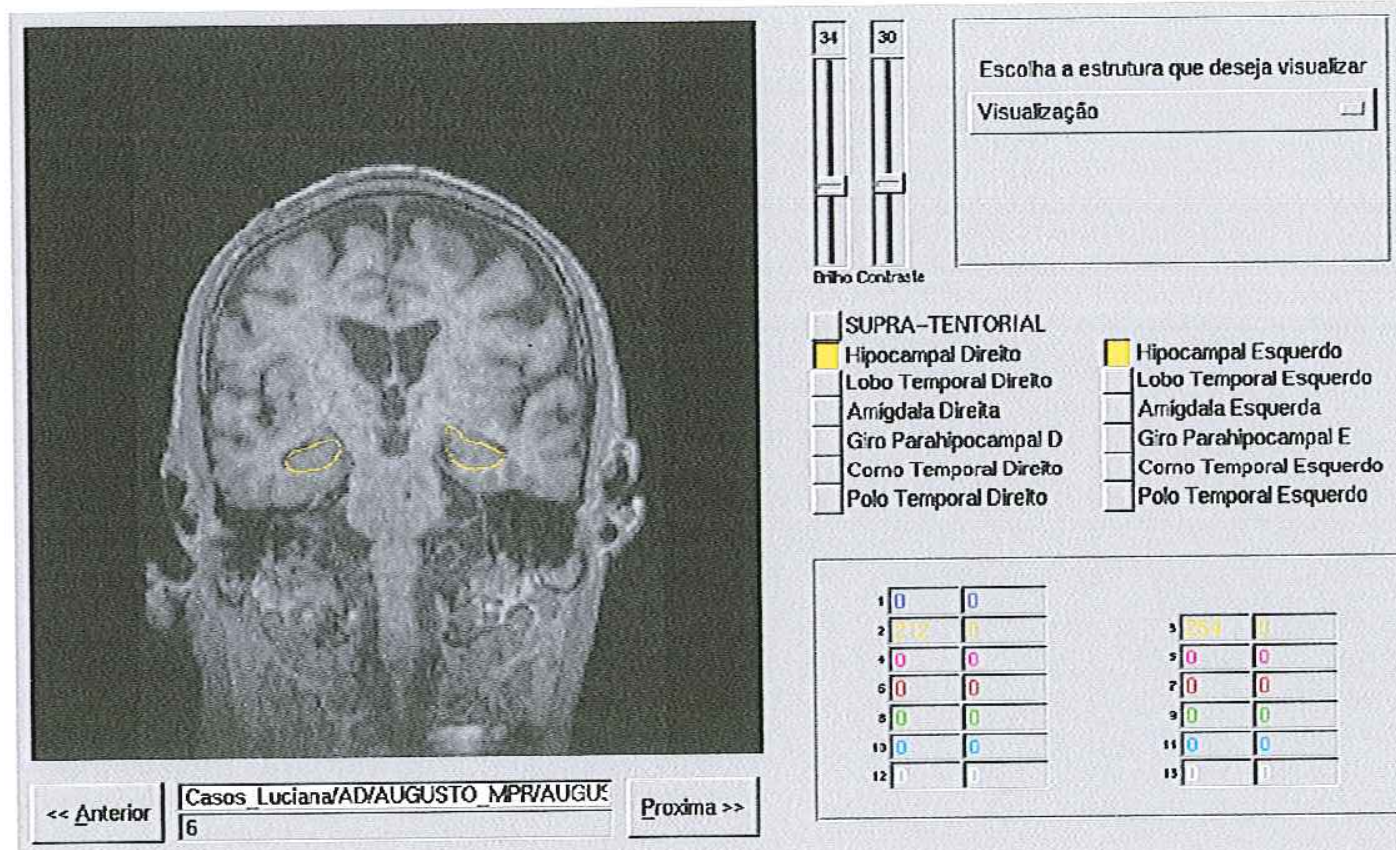

JSUPRA-TENTORIAL

Hipocampal Direito

$\Gamma$ Hipocampal Esquerdo

Lobo Temporal Diretio

Amigdala Direk̂a

Lobo Temporal Esquerdo

Giro Parahipocampal D

Amigdala Esquerda

Corno Temporal Direto

Giro Parahipocampal E

Polo Temporal Direlto

Corno Temporal Esquerdo
Polo Temporal Esquerdo
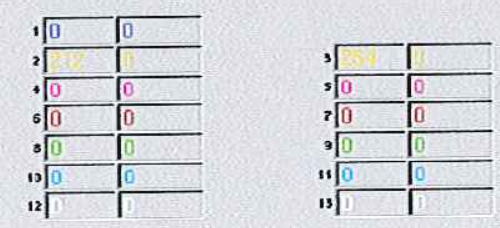

Figura 5.4. Interface com algumas estruturas de interesse marcadas.

\subsection{Avaliação qualitativa}

As interfaces foram desenvolvidas de modo a se tentar facilitar ao máximo

o trabalho dos médicos, tendo sido avaliadas por dois especialistas segundo os critérios mostrados na Tabela 5.1. 


\begin{tabular}{|l|c|c|c|c|}
\hline \multicolumn{1}{|c|}{ Usuários } & $\begin{array}{c}\text { Facilidade de } \\
\text { Uso }\end{array}$ & $\begin{array}{c}\text { Apresentação } \\
\text { Geral }\end{array}$ & $\begin{array}{c}\text { Resultados } \\
\text { Obtidos }\end{array}$ & $\begin{array}{c}\text { Comparativo } \\
\text { com Scion }\end{array}$ \\
\hline Usuário 1 & 3 & 4 & 4 & 4 \\
\hline Usuário 2 & 4 & 3 & 4 & 4 \\
\hline Valores & 1-Ruim & 2-Regular & 3 -Bom & 4-Excelente \\
\hline
\end{tabular}

Tabela 5.1 - Valores para a avaliação da interaface

O tempo gasto para marcar uma média de 8 estruturas em cada imagem do estudo é de aproximadamente 3 minutos. O tempo total para obtenção dos resultados varia de acordo com a quantidade de imagens pertencentes ao estudo.

\subsection{Avaliação quantitativa}

A experiência prova que nunca se obtém resultados iguais ao refazer as marcações das estruturas de um paciente, visto que há sempre variações intraespecialista e inter-especialista a serem levadas em consideração quando da segmentação manual das estruturas. Segundo os especialistas do Hospital das Clínicas uma variação de até 1,5 milímetros cúbicos é considerada uma medição muito boa. A tabela 5.3 apresenta os resultados obtidos em três casos e a correlação entre os valores obtidos através do Scion e a ferramenta desenvolvida. 


\begin{tabular}{|l|r|r|r|r|r|r|r|r|r|}
\hline \multirow{2}{*}{ Paciente } & \multicolumn{3}{|c|}{ Valores Anteriores } & \multicolumn{3}{c|}{ Valores Obtidos } & \multicolumn{3}{|c|}{ Diferença (\%) } \\
\cline { 2 - 11 } & HD & HE & Encéfalo & \multicolumn{1}{l|}{ HD } & HE & Encéfalo & HD & HE & Encéfalo \\
\hline Caso 1 & 2,43 & 2,15 & 307,56 & 2,4 & 2,6 & 336 & 1,25 & 2,09 & 0,92 \\
\hline Caso 2 & 1,34 & 1,32 & 270,5 & 1,13 & 0,81 & 269,39 & 1,8 & 6,29 & 0,41 \\
\hline Caso 3 & 1,58 & 1,82 & 232,47 & 1,21 & 1,05 & 332,96 & 3,05 & 6,85 & 4,32 \\
\hline
\end{tabular}

Tabela 5.2 - Resultados comparativos obtidos através do sistema em $\mathrm{mm}^{3}$. 


\section{Capítulo 6}

\section{Discussão dos Resultados e Conclusões}

De modo geral, os valores obtidos pelo sistema enquadram-se dentro da variação quantitativa considerada aceitável, destacando-se porém, que o tempo médio gasto para obtenção dos resultados de um estudo foi de aproximadamente 25 minutos, variando de acordo com a quantidade de imagens. Esta redução no tempo médio para obtenção dos resultados ajuda muito os profissionais da área, visto que com a utilização do Scion o tempo médio era de 1 hora e meia e com uma margem de erro muito grande, visto que a cada estrutura marcada o especialista precisava copiar o resultado apresentado para uma planilha eletrônica.

Visto que um dos objetivos principais do desenvolvimento do projeto foi a implementação de uma ferramenta rápida, precisa e eficaz na função a que se destina, os resultados comprovaram que não há mais a necessidade de utilização de software de terceiros junto à rotina do CIREP, pois esta ferramenta supre as necessidades dos especialistas que trabalham com Mal de Alzheimer e Epilepsia e que necessitam de medidas volumétricas.

Além disso, com o desenvolvimento de uma ferramenta open-source, trabalhando sob a plataforma de um sistema operacional gratuito, todos os especialistas 
do Hospital das Clínicas poderão ter acesso à ferramenta e utiliza-la na rotina de diagnóstico de pacientes suspeitos de Epilepsia e Mal de Alzheimer, não causando custos com aquisição de softwares prontos ou licenças de uso adicionais. Deve-se destacar também que o desenvolvimento de uma ferramenta deste tipo possibilita alto grau de aplicabilidade e atualização, pois possui seu código fonte aberto, podendo ser alterado a qualquer momento de acordo com as necessidades dos especialistas, podem ser agregados novos módulos e funcionalidades, sem custos adicionais.

Uma das principais preocupações no desenvolvimento da ferramenta foi quanto à interface a ser apresentada aos usuários, pois precisa ser de fácil utilização e manter uma performance aceitável. Partindo desse princípio foi desenvolvida de acordo com padrões de layout intuitivos e cores visualmente não agressivas, com isso a ferramenta tornando-se de fácil treinamento e fácil interação. O que deve reduzir o tempo necessário para treinar os profissionais que serão usuários do sistema.

Enquadram-se dentro das principais características da ferramenta a navegabilidade entre as imagens ordenadas com estruturas já marcadas, o armazenamento e visualização de estudos já realizados e a possibilidade de remarcar apenas algumas estruturas de interesse sem que haja a necessidade de remarcar todas as estruturas anteriormente definidas. Para cada estudo realizado pela ferramenta é criado um diretório com o nome do estudo, um arquivo contendo o cabeçalho da imagem e a seqüência ordenada destas, arquivos que armazenam as coordenadas $\mathrm{X}$ e $\mathrm{Y}$ dos pontos de cada estrutura segmentada em cada imagem e, finalmente o resultado do estudo também é armazenado.

A ferramenta possui um certo grau de automação, visto que quando o 
médico especialista marca as estruturas de interesse em uma imagem, os pontos marcados para cada estrutura são redesenhados sobre a próxima imagem. A grande vantagem disso é que algumas estruturas não possuem diferenças significativas ao serem marcadas de uma imagem para outra, poupando o tempo que o médico especialista gastaria para refazer a marcação e reduzindo a margem de erro. Caso a marcação deva ser refeita, a ferramenta de segmentação manual possibilita apagar esta pré-marcação e refaze-la sem perda das outras marcações efetuadas na mesma imagem.

Outro aspecto importante é que, apesar de a ferramenta ter sido desenvolvida para cálculo volumétrico de estruturas cerebrais, poderão ser quantificadas imagens de outras regiões anatômicas, desde que sejam imagens de Ressonância Magnética Nuclear no padrão DICOM 3.0.

Deve-se ressaltar que o algoritmo é um dos módulos que compõem uma ferramenta de auxílio ao diagnóstico em neurologia que está sendo desenvolvida para ser utilizada pelos especialistas do Hospital das Clínicas da Faculdade de Medicina de Ribeirão Preto. As imagens marcadas no módulo de volumetria podem ser reconstruídas tri-dimensionalmente através do módulo de reconstrução 3D. As imagens e as coordenadas das estruturas são interconectadas, assim pode-se separar as estruturas das imagens e reconstruí-las em três dimensões (Figura 6.1). Existe também um módulo de co-registro de imagens (Ressonância Magnética, SPECT e Tomografia Computadorizada) que utiliza imagens de exames complementares, no qual o especialista posiciona marcadores e seus respectivos pares nas duas imagens, possibilitando seu co-registro e fusão (Figura 6.2). Estes três módulos auxiliam na localização ou na quantização de lesões cerebrais. A integração final destes três 
sistema de auxílio ao diagnóstico em neurologia contendo as ferramentas básicas necessárias, de baixo custo e fácil utilização. Deve-se ressaltar que a utilização de uma plataforma open-source torna a ferramenta desenvolvida extremamente flexível, podendo incorporar novas funções e aplicativos.

Finalmente, como indicação de trabalhos futuros, seguindo a mesma linha de pesquisa, seria de grande valia se as informações das marcações fossem armazenadas em um banco de dados para melhor avaliação, recuperação e geração de dados estatísticos para o CIREP. Quando temos informações armazenadas em um banco de dados possuímos várias maneiras de quantifica-las e reproduzir os mais diversos resultados comparativos para o usuário, ao passo que da forma que está implementado, as informações não ficam centralizadas.

Outra linha de investigação seria o desenvolvimento de um algoritmo computacional para realizar a segmentação e volumetria automatizadas das estruturas cerebrais.

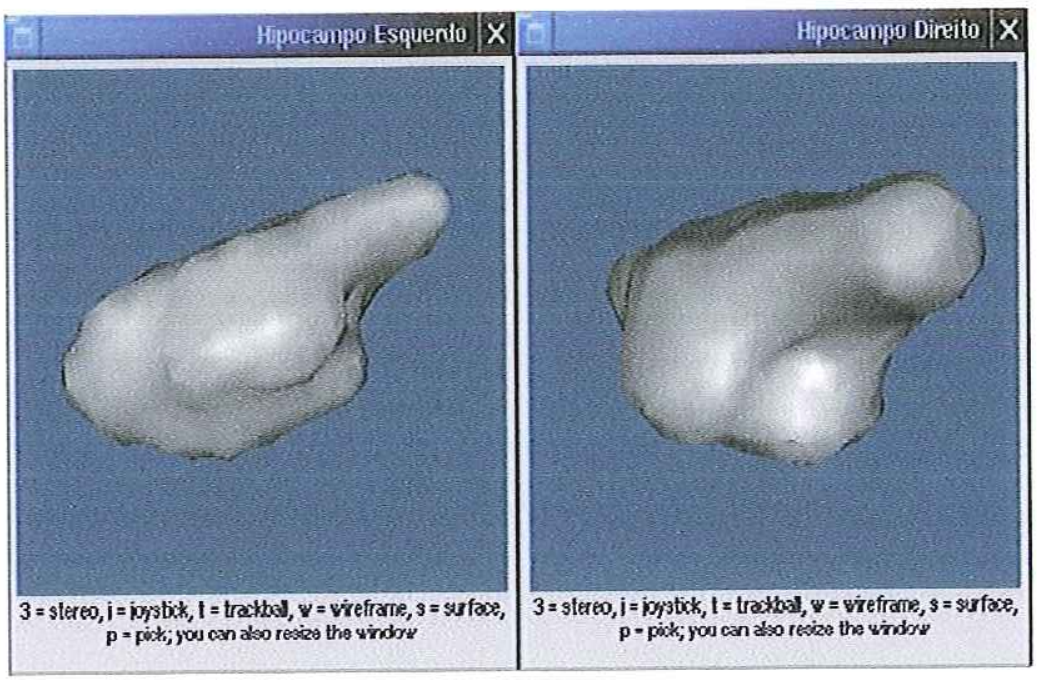

Figura 6.1 - Reconstrução do Hipocampo 


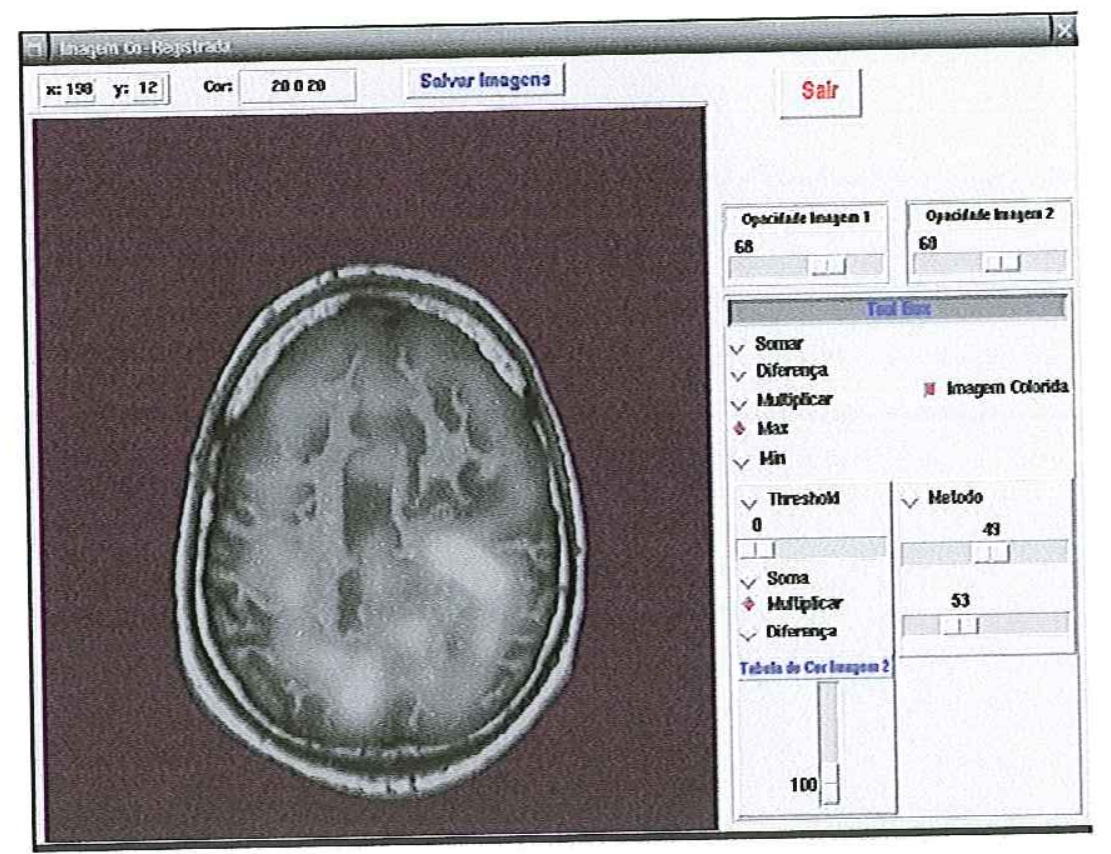

Figura 6.2-Módulo de Co-registro: Imagem de RM e de SPECT com marcadores posicionados. 
Capítulo 7

Referências Bibliográficas

[ABRAHÃO-2000]

ABRAHÃ̃, M. T.; AMORIN, M. F. A Utilização do HL7 na Comunicą̧ão de Sistemas de Informação em Saúde. Anais do CBEB'2000.

[BALLESTER-2000]

BALLESTER, M. Á. G. ; ZISSERMAN, A. ; BRADY, M. Segmentation and measurement of brain structures in MRI including confidence bounds. Medical Image Analysis 4(2000) 189-200.

\section{[BIDGOOD-1998]}

BIDGOOD JR., W. D. The SNOMED DICOM Microglossary: Controlled Terminology Resource for Data Interchange in Biomedical Imaging. Meth Inform Med $1998 ; 37: 404-414$.

[CARITÁ-2000]

CARITÁ, E. C. Vinculação de Imagens para Busca e Visualização a partir de Sistema de Informação Radiológica (RIS). CBIS 2000.

[CENDES-1995]

CENDES, F. et al. Frequency and characteristics of dual pathology in patients with lesional epilepsy. Neurology 45:2058 - 2064, 1995.

\section{[CIESIELSKI-1999]}

CIESIELSKI,K.T. et al. MRI Morphometry of Mamillary Bodies, Caudate Nuclei, and Prefrontral Cortices After Chemotherapy of Chilhood Leukemia: Multivariate Models of Early an Late Developing Memory Subsystems. Behavioral Neurosciense Vol. 113, No. 3,439-450, 1999.

[COOK-1992]

COOK,M.J. et al. Hippocampal Volumetric and Morphometric Studies in Frontal and Tempral Lobe Epilepsy. Brain 115:1001-1015, 1992. 


\section{[ELIEZ-2000]}

ELIEZ, S. et al. Children and Adolescents with Velocardiofacial Syndrome: a volumetric MRI study. Am J Psychiatry 157:3, March 2000.

[FREIRE-1997]

FREIRE, H. J. P. Proposta de um Sistema de Banco de Dados para Tomografia por Ressonância Magnética Nuclear. São Carlos: 1997.

[FOX-2000]

FOX, N. C. et al. Using Serial Registered Brain Magnetic Resonance Imaging to Measure Disease Progression in Alzheimer Disease: Power Calculations and Estimates of Sample Size to Detect Treatment Effects. Arch Neurol. Vol. 57, Mar 2000.

[HOGAN-2000]

HOGAN, R. E. et al. Mesial Temporal Sclerosis and Temporal Lobe Epilepsy: MR Imaging Deformation-based Segmentation of the Hippocampus in Five Patientes. Radiology, 2000; 216: 291-297.

[INSAUSTI-1998]

INSAUSTI, R. et al. MR Volumetric Analysis of the human Entorhinal, Perirhinal e Temporopolar Cortices. AJNR Am J Neuroradiol 19:659-671, April 1998.

[JACK JR.-1992b]

JACK JR., C. R. et al. Magnetic Resonance Image-Based Hippocampal Volumetry: Correlation with Outcome after Temporal Lobectomy. American Neurological Association, 1992, vol. 31, no. 2, pp. 138-146.

[JACK JR.-1992a]

JACK JR., C. R. et al. MR-based hippocampal volumetry in the diagnosis of Alzheimer's disease. Neurology, (42) Jan. 1992, pp. 183-188.

[KUZNIECKY-1997]

KUZNIECKY, R. I. et al. Multimodality MRI in mesial temporal sclerosis: Relative sensitivity and specificity. Neurology 1997; 49: pp. 774-778.

[LAAKSO-1997]

LAAKSO, M. P. et al. MRI Volumetry of the Hippocampus: the effect of slice thickness on volume formation. Magnetic Resonance Imaging, Vol. 15, no. 2, pp. 263-265, 1997.

[LAWSON-1997]

LAWSON, J. A. et al. Quantitative MRI in Outpatient Childhood Epilepsy. International League Against Epilepsy. 38(12):1289-1293 1997.

[LEMIEUX-2000]

LEMIEUX, L. et al. Hippocampal and cerebellar volumetry in serially acquired MRI volume scans. Magnetic Resonance Imaging 18:1027-1033, 2000. 
[MIZUNO-2000]

MIZUNO, K. et al. Medial temporal atrophy and memory impairment in early stage of Alzheimer's disease: an MRI volumetric and memory assessment study. Journal of the Neurological Sciences. 173: 18-24, 2000.

[NEMA-1999]

NEMA (National Electrical Manufacturers Association). Digital Imaging and Communications in Medicine (DICOM). Virginia, 1999.

[OOSERWIJK]

OOSERWIJK, H. DICOM versus HL7 for Modality Interfacing. Journal of Digital Imaging, Vol. 11, no. 3, Suppl. 1 August, 1998: pp. 39-41.

[PISA-2000]

PISA, I. T.; RUIZ, E. E. S. Proposta de Utilização da Arquitetura CORBA para o Desenvolvimento de um PACS Distribuido. CBIS 2000.

[REVET-1997]

REVET, B. DICOM Cook Book for Implementations in Modalities. Philips Medical Systems Nederland B. V., 1997.

[ROGACHESKI-1998]

ROGACHESKI, $\hat{\mathbf{E}}$. et al. Análises visual e volumétrica por ressonância magnética das formações hipocampais em um grupo de pacientes com diagnóstico clínico de epilepsia do lobo temporal. Arq Neuropsiquiatr 1998; 56(3-A): 419-428.

[ROVARIS-1999]

ROVARIS, M. et al. Reproducibility of Brain in MRI Lesion Volume Measurements in Multiple Sclerosis Using a Local thresholding technique: effects of formal operator training. Eur Neurol 1999; 41:226-230.

[SANTOS-2000]

SANTOS, M.; RUIZ, E. E. S. Implementação de Módulos para Comunicação de Imagens Médicas em Protocolo DICOM. CBIS 2000

[SCHROEDER-2000]

SCHROEDER, W. et al. The VTK User's Guide. Kitware, 2000.

[SIEGEL-1999]

SIEGEL, E. L.; KOLODNER, R. M. FilmlessRadiology. New York: Springer-Verlag, 1999.

[STASIU-2000]

STASIU, R. K.; BICHINHO, G. L. Proposta de um Modelo de Objetos para Informações Armazenadas em Arquivos DICOM. CBIS 2000. 
[SUSAN-1998]

SUSAN, S. H.; KUZNIECKY, R. L. Fundamentos Neurobiológicos das Epilepsias: Aspectos Clínicos e Cirúrgicos, vol. I, 1998.

[SWEET-2000]

SWEET, M.; EARLS, C. P.; SPITZAK, B. FLTK 1.0.10 Programming Manual. Revision 16, 2000.

[WATSON-1995]

WATSON, C.; WILLIAMSON, B. Volumetric magnetic resonance imaging in patients with primary generalized epilepsy. J. Epilepsy 8:104-109, 1995.

[WATSON-1996]

WATSON, C. et al. Volumetric magnetic resonance imaging in patients with secondary generalized epilepsy. J. Epilepsy 9:14-19, 1996.

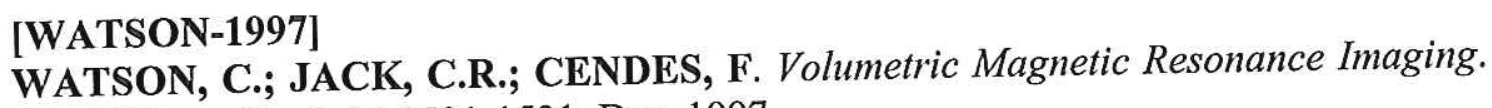
ARCH Neurol/vol. 54:1521-1531, Dec. 1997.

[WATSON-1998]

WATSON, C.; CENDES, F. Fundamentos Neurobiológicos das Epilepsias: Aspectos Clínicos e Cirúrgicos, vol. I, 1998.

[WEBB-1999]

WEBB, J. et al. Automatic detection of hippocampal atrophy on magnetic resonance images. Magnetic Resonance Imaging, Vol. 17, No. 8, pp. 1149-1161, 1999.

[WHITWELL-2001]

Whitwell, J. L. et al. Normalization of Cerebral Volumes by Use of Intracranial Volume: Implications for Longitudinal Quantitative MR Imaging. AJNR, vol. 22:14831489, Set. 2001. 
ANEXOS 


\begin{tabular}{|c|c|c|c|c|c|c|c|c|c|c|c|c|c|c|}
\hline & 1 & 2 & 3 & 4 & 5 & 6 & 7 & 8 & 9 & 10 & 11 & 12 & 13 & 14. \\
\hline PTD & 1,5 & 1,86 & 2,77 & 6,07 & 7,1 & 8,04 & 8,09 & 8,54 & 9,99 & 10,58 & & & & \\
\hline PTE & 0,64 & 2,31 & 2,86 & 4,53 & 5,07 & 6,31 & 7,26 & 8,3 & 10,37 & 9,72 & 10,63 & & & \\
\hline$\angle T D$ & 11,16 & 12,24 & 12,56 & 13,49 & 13,21 & 13,15 & 13,1 & 12,67 & 12,23 & 12,24 & 11,84 & 11,51 & 11,83 & 12,5 \\
\hline LTE & 11,12 & 12,25 & 12,01 & 13,32 & 13,3 & 13,11 & 13,35 & 13 & 12,28 & 12,16 & 11,91 & 12,26 & 12,52 & 13 \\
\hline AMD & 1,03 & 1,37 & 1,31 & 1,32 & 1,35 & 1,45 & 1,43 & 1,28 & & & & & & \\
\hline AME & 1,1 & 1,63 & 1,73 & 1,31 & 1,6 & 1,39 & 1,61 & 0,92 & & & & & & \\
\hline$H D$ & 0,82 & 0,95 & 1,21 & 1,06 & 1,01 & 1,38 & 1,28 & 0,78 & 0,77 & 0,73 & 0,63 & 0,66 & 0,65 & 0,68 \\
\hline$H E$ & 0,39 & 0,77 & 0,66 & 0,71 & 0,74 & 0,75 & 0,49 & 0,58 & 0,52 & 0,45 & 0,46 & 0,53 & 0,45 & 0,38 \\
\hline CED & 0,59 & 0,47 & 0,44 & 0,44 & 0,37 & 0,29 & 0,3 & 0,35 & 0,35 & 0,32 & 0,25 & 0,31 & & \\
\hline CEE & 0,57 & 0,57 & 0,5 & 0,34 & 0,38 & 0,29 & 0,26 & 0.49 & 0,26 & 0,39 & 0,39 & 0,44 & & \\
\hline CTVD & 0,08 & 0,13 & 0,15 & 0,14 & 0.26 & 0,2 & 0,12 & 0,04 & & & & & & \\
\hline CTVE & 0,08 & 0,16 & 0,21 & 0,28 & 0,34 & 0,46 & 0,17 & 0,19 & 0,08 & & & & & \\
\hline VST & 88,53 & 90,82 & 91,79 & 91,82 & 94,62 & 98,03 & 95,58 & 96,54 & 96,1 & 95,81 & 98,04 & 97,56 & 98,13 & 98,58 \\
\hline
\end{tabular}

\begin{tabular}{|c|c|c|c|c|c|c|c|c|c|c|c|c|c|}
\hline & LT & & AM & & HIP & & CE & & CTV & & PT & & VST \\
\hline ASSIMETRIA & $-0,96688$ & & $-6,87128$ & & 43,74752 & & $-8,54701$ & & $-55,0162$ & & $-5,22107$ & & \\
\hline \multirow[b]{2}{*}{ ABSOLUTO } & $\bar{D}$ & E & D & E & $\mathrm{D}$ & E & $\mathrm{D}$ & E & $\bar{D}$ & E & $\bar{D}$ & E & 344,814 \\
\hline & 4961 & 50,092 & 2.108 & 2258 & 30 & 1968 & 0.896 & 0976 & 0.224 & 0,394 & 12,908 & 136 & \\
\hline \multirow[b]{2}{*}{ NORMALIZADO } & $\mathrm{D}$ & E & $\mathrm{D}$ & E & $\mathrm{D}$ & E & $\mathrm{D}$ & E & $\bar{D}$ & EE & D & E & \\
\hline & 14.38747 & $\sqrt{145250}$ & 0.611344 & 0,654346 & 0.89033 & 0580742 & 0,25985 & 0.2833051 & 0,064963 & 0,112265 & 3.743467 & 3.944155 & \\
\hline
\end{tabular}




\begin{tabular}{|r|r|r|r|r|r|r|r|r|r|r|r|r|r|}
\hline 15 & 16 & 17 & 18 & 19 & 20 & 21 & 22 & 23 & 24 & 25 & 26 & vol.total & indice de assimetria \\
\hline & & & & & & & & & & & & 12,908 & $-5,221065339$ \\
\hline & & & & & & & & & & & & 13,6 & \\
\hline 13,53 & 14,16 & 14,95 & 15,08 & 16,6 & & & & & & & & 49,61 & $-0,966881306$ \\
\hline 13,56 & 15 & 15,01 & 15,59 & 15,71 & & & & & & & & 50,092 & \\
\hline & & & & & & & & & & & & 2,108 & $-6,871278058$ \\
\hline & & & & & & & & & & & & 2,258 & \\
\hline 0,65 & 0,66 & 0,66 & 0,77 & & & & & & & & & 3,07 & 43,74751886 \\
\hline 0,5 & 0,5 & 0,49 & 0,47 & & & & & & & & & 1,968 & \\
\hline & & & & & & & & & & & & 0,896 & $-8,547008547$ \\
\hline & & & & & & & & & & & 0,976 & \\
\hline & & & & & & & & & & & 0,224 & $-55,01618123$ \\
\hline & & & & & & & & & & & 0,394 & \\
\hline
\end{tabular}

